\title{
EQUIVARIANT GEOMETRY OF BANACH SPACES AND TOPOLOGICAL GROUPS
}

\author{
CHRISTIAN ROSENDAL \\ Department of Mathematics, Statistics, and Computer Science (M/C 249), \\ University of Illinois at Chicago, 851 S. Morgan St., Chicago, IL 60607-7045, USA; \\ email: rosendal.math@gmail.com
}

Received 4 October 2016; accepted 9 August 2017

\begin{abstract}
We study uniform and coarse embeddings between Banach spaces and topological groups. A particular focus is put on equivariant embeddings, that is, continuous cocycles associated to continuous affine isometric actions of topological groups on separable Banach spaces with varying geometry.
\end{abstract}

2010 Mathematics Subject Classification: 46B80, 46B20 (primary); 20F65 (secondary)

\section{Introduction}

The present paper is a contribution to the study of large scale geometry of Banach spaces and topological groups and, in particular, to questions of embeddability between these objects. In a sense, our aim is somewhat wider than usual as we will be dealing with general Polish groups as opposed to only locally compact groups. This is achieved by using the recently developed framework of [57], which allows us to treat Banach spaces and topological groups under one heading. Still our focus will be restricted as we are mainly interested in equivariant maps, that is, cocycles associated to affine isometric actions on Banach spaces.

To state the results of the paper, let us begin by fixing the basic terminology. Given a map $\sigma:(X, d) \rightarrow(Y, \partial)$ between metric spaces, we define the compression modulus by

$$
\kappa_{\sigma}(t)=\inf (\partial(\sigma(a), \sigma(b)) \mid d(a, b) \geqslant t)
$$

(c) The Author 2017. This is an Open Access article, distributed under the terms of the Creative Commons Attribution licence (http://creativecommons.org/licenses/by/4.0/), which permits unrestricted re-use, distribution, and reproduction in any medium, provided the original work is properly cited. 
and the expansion modulus by

$$
\theta_{\sigma}(t)=\sup (\partial(\sigma(a), \sigma(b)) \mid d(a, b) \leqslant t) .
$$

Thus $\sigma$ is uniformly continuous if $\lim _{t \rightarrow 0_{+}} \theta_{\sigma}(t)=0$ (in which case $\theta_{\sigma}$ becomes the modulus of uniform continuity) and a uniform embedding if, moreover, $\kappa_{\sigma}(t)>0$ for all $t>0$. Furthermore, $\sigma$ is bornologous if $\theta_{\sigma}(t)<\infty$ for all $t<\infty$ and expanding if $\lim _{t \rightarrow \infty} \kappa_{\sigma}(t)=\infty$. A bornologous expanding map is called a coarse embedding. We also define $\sigma$ to be uncollapsed if $\kappa_{\sigma}(t)>0$ for just some sufficiently large $t>0$.

As we will be studying uniform and coarse embeddability between Banach spaces and topological groups, we must extend the above concepts to the larger categories of uniform and coarse spaces and also show how every topological group is canonically equipped with both a uniform and a coarse structure.

Postponing for the moment this discussion, let consider the outcomes of our study. As pointed out by Kalton [35], the concepts of uniform and coarse embeddability between Banach spaces seem very tightly related. Though, Kalton [36] eventually was able to give an example of two separable Banach spaces that are coarsely equivalent, but not uniformly homeomorphic, the following basic question of Kalton concerning embeddings remains open.

QUESTION 1. Does the following equivalence hold for all (separable) Banach spaces?

$X$ is uniformly embeddable into $E \Longleftrightarrow X$ is coarsely embeddable into $E$.

Relying on entirely elementary techniques, our first result shows that in many settings we do have an implication from left to right.

THEOREM 1. Suppose $\sigma: X \rightarrow E$ is an uncollapsed uniformly continuous map between Banach spaces. Then, for any $1 \leqslant p<\infty, X$ admits a simultaneously uniform and coarse embedding into $\ell^{p}(E)$.

Since both uniform and coarse embeddings are uncollapsed, we see that if $X$ is uniformly embeddable into $E$, then $X$ is coarsely embeddable into $\ell^{p}(E)$. For the other direction, if $X$ admits a uniformly continuous coarse embedding into $E$, then $X$ is uniformly embeddable into $\ell^{p}(E)$. It is therefore natural to ask to which extent bornologous maps can be replaced by uniformly continuous maps. In particular, is every bornologous map $\sigma: X \rightarrow E$ between Banach spaces close to a uniformly continuous map, that is, is there a uniformly continuous map $\tilde{\sigma}$ so that $\sup _{x \in X}\|\sigma(x)-\tilde{\sigma}(x)\|<\infty$ ? 
As it turns out, Naor [46] was recently able to answer our question in the negative, namely, there are separable Banach spaces $X$ and $E$ and a bornologous map between them which is not close to any uniformly continuous map. Nevertheless, several weaker questions remain open.

Also, in case the passage from $E$ to $\ell^{p}(E)$ proves troublesome, we can get by with $E \oplus E$ under stronger assumptions.

THEOREM 2. Suppose $\sigma: X \rightarrow B_{E}$ is an uncollapsed uniformly continuous map from a Banach space $X$ into the ball of a Banach space $E$. Then $X$ admits a uniformly continuous coarse embedding into $E \oplus E$.

For example, if $X$ is a Banach space uniformly embeddable into its unit ball $B_{X}$, for example, if $X=\ell^{2}$, then, whenever $X$ uniformly embeds into a Banach space $E$, it coarsely embeds into $E \oplus E$.

The main aim of the present paper, however, is to consider equivariant embeddings between topological groups and Banach spaces, that is, continuous cocycles. So let $\pi: G \curvearrowright E$ be a strongly continuous isometric linear representation of a topological group $G$ on a Banach space $E$, that is, each $\pi(g)$ is a linear isometry of $E$ and, for every $\xi \in E$, the map $g \in G \mapsto \pi(g) \xi$ is continuous. A continuous cocycle associated to $\pi$ is a continuous map $b: G \rightarrow E$ satisfying the cocycle equation

$$
b(g f)=\pi(g) b(f)+b(g) .
$$

This corresponds to the requirement that $\alpha(g) \xi=\pi(g) \xi+b(g)$ defines a continuous action of $G$ by affine isometries on $E$. As $b$ is simply the orbit map $g \mapsto \alpha(g) 0$, it follows that continuous cocycles are actually uniformly continuous and bornologous. We call a continuous cocycle $b: G \rightarrow E$ coarsely proper if it is a coarse embedding of $G$ into $E$. In this case, we also say that the associated affine isometric action $\alpha$ is coarsely proper.

For the next result, a topological group $G$ has the Haagerup property if it has a strongly continuous unitary representation with an associated coarsely proper cocycle. Though it is known that every locally compact second countable amenable group has the Haagerup property [8], this is very far from being true for general topological groups. Indeed, even for Polish groups, that is, separable completely metrizable topological groups, such as separable Banach spaces, this is a significant requirement.

Building on work of Aharoni et al. [3], we show the following equivalence.

THEOREM 3. The following conditions are equivalent for an amenable Polish group $G$ :

(1) G coarsely embeds into a Hilbert space;

(2) G has the Haagerup property. 
Restricting to Banach spaces, we have a stronger result, where the equivalence of (1) and (2) is due to Randrianarivony [51].

THEOREM 4. The following conditions are equivalent for a separable Banach space $X$ :

(1) X coarsely embeds into a Hilbert space;

(2) X uniformly embeds into a Hilbert space;

(3) X admits an uncollapsed uniformly continuous map into a Hilbert space;

(4) X has the Haagerup property.

Even from collapsed maps we may obtain information, provided that there is just some single distance not entirely collapsed.

TheOrem 5. Suppose $\sigma: X \rightarrow \mathcal{H}$ is a uniformly continuous map from a separable Banach space into Hilbert space so that, for some single $r>0$,

$$
\inf _{\|x-y\|=r}\|\sigma(x)-\sigma(y)\|>0 .
$$

Then $B_{X}$ uniformly embeds into $B_{\mathcal{H}}$.

One of the motivations for studying cocycles, as opposed to general uniform or coarse embeddings, is that cocycles are also algebraic maps, that is, reflect algebraic features of the acting group $G$. As such, they have a higher degree of regularity and permit us to carry geometric information from the phase space back to the acting group. For example, a continuous uncollapsed cocycle between Banach spaces is automatically a uniform embedding. Similarly, a cocycle $b: X \rightarrow E$ between Banach spaces associated to the trivial representation $\pi \equiv \mathrm{id}_{E}$ is simply a bounded linear operator, so a cocycle may be considered second best to a linear operator. We shall encounter and exploit many more instances of this added regularity throughout the paper.

Relaxing the geometric restrictions on the phase space, the next case to consider is that of super-reflexive spaces, that is, spaces admitting a uniformly convex renorming. For this class, earlier work was done by Pestov [49] and Naor and Peres [47] for discrete amenable groups $G$. For topological groups, several severe obstructions appear and it does not seem possible to get an exact analogue of Theorem 3. Indeed, the very concept of amenability requires re-examination. We say that a Polish group $G$ is Folner amenable if there is either a continuous homomorphism $\phi: H \rightarrow G$ from a locally compact second countable amenable 
group $H$ with dense image or if $G$ admits a chain of compact subgroups with dense union. For example, a separable Banach space is Følner amenable.

THEOREM 6. Let $G$ be a Følner amenable Polish group admitting a uniformly continuous coarse embedding into a Banach space E. Then, for every $1 \leqslant p<\infty$, $G$ admits a coarsely proper continuous affine isometric action on a Banach space $V$ that is finitely representable in $L^{p}(E)$.

We note that, if $E$ is super-reflexive, then so is every $V$ finitely representable in $L^{2}(E)$. Again, for Banach spaces, this leads to the following.

THEOREM 7. Suppose $X$ is a separable Banach space admitting an uncollapsed uniformly continuous map into a super-reflexive space. Then $X$ admits a coarsely proper continuous cocycle with values in a super-reflexive space.

For our next results, we define a topological group to be metrically stable if it admits a compatible left-invariant stable metric. By results of $[10,41,60]$, a Polish group is metrically stable if and only if it is isomorphic to a subgroup of the linear isometry group of a separable reflexive Banach space under the strong operator topology. Also, by a result of Raynaud [52], a metrically stable Banach space contains a copy of $\ell^{p}$ for some $1 \leqslant p<\infty$.

Using the construction underlying Theorem 6 , we obtain information on spaces uniformly embeddable into balls of super-reflexive spaces.

THEOREM 8. Let $X$ be a Banach space admitting an uncollapsed uniformly continuous map into the unit ball $B_{E}$ of a super-reflexive Banach space $E$. Then $X$ is metrically stable and contains an isomorphic copy of $\ell^{p}$ for some $1 \leqslant p<\infty$.

As an application, this restricts the class of super-reflexive spaces uniformly embeddable into their own balls.

For a general nonamenable topological group $G$, we may also produce coarsely proper affine isometric actions on reflexive spaces starting directly from a stable metric or écart.

THEOREM 9. Suppose a topological group G carries a continuous left-invariant coarsely proper stable écart. Then $G$ admits a coarsely proper continuous affine isometric action on a reflexive Banach space.

Intended applications here are, for example, automorphism groups of countable atomic models of countable stable first-order theories that, under the additional 
assumption of being locally (OB), satisfy the conditions of Theorem 9. Note that Theorem 9 may also be viewed as a generalization of a result of Haagerup and Przybyszewska [30] stating that every locally compact second countable group admits a proper affine isometric action on a reflexive space. Indeed, every locally compact second countable group admits a compatible proper left-invariant metric, which by properness is automatically stable.

The final part of our investigations concern a fixed point property for affine isometric group actions. We identify a geometric incompatibility between a topological group $G$ and a Banach space $E$ strong enough to ensure that not only does $G$ have no coarsely proper affine isometric action on $E$, but every affine isometric action even has a fixed point.

The two main concepts here are solvent maps and geometric Gelfand pairs. First, a map $\phi: X \rightarrow Y$ between metric spaces is solvent if, for every $n$, there is an $R$ with $R \leqslant d\left(x, x^{\prime}\right) \leqslant R+n \Rightarrow d\left(\phi x, \phi x^{\prime}\right) \geqslant n$. Refining earlier results of Kalton, we show that every bornologous map from $c_{0}$ to a reflexive Banach space is insolvent. Also a coarsely proper continuous isometric action $G \curvearrowright X$ of a topological group $G$ on a metric space $X$ is said to be a geometric Gelfand pair if, for some $K$ and all $x, y, z, u \in X$ with $d(x, y) \leqslant d(z, u)$, there is $g \in G$ so that $d(g(x), z) \leqslant K$ and $d(z, g(y))+d(g(y), u) \leqslant d(z, u)+K$. This second condition is typically verified when $X$ is sufficiently geodesic and the action of $G$ is almost doubly transitive. For example, if $\operatorname{Aff}(X)$ denotes the group of affine isometries of a Banach space $X$, then $\operatorname{Aff}(X) \curvearrowright X$ is a geometric Gelfand pair when $X=L^{p}([0,1]), 1 \leqslant p<\infty$, and when $X$ is the Gurarii space. Similarly, if $X$ is the integral, $\mathbb{Z} \mathbb{U}$, or rational, $\mathbb{Q U}$, Urysohn metric space, then $\operatorname{Isom}(X) \curvearrowright X$ is a geometric Gelfand pair.

THEOREM 10. Suppose $G \curvearrowright X$ is a geometric Gelfand pair and $Y$ is a metric space so that every bornologous map $X \rightarrow Y$ is insolvent. Then every continuous isometric action $G \curvearrowright Y$ has bounded orbits.

Combining Theorem 10 with the observations above and the fixed point theorems of [58] and [6], we obtain the following corollary.

COROLLARY 1. Every continuous affine isometric action of $\operatorname{Isom}(\mathbb{Q U})$ on a reflexive Banach space or on $L^{1}([0,1])$ has a fixed point.

\section{Uniform and coarse structures on topological groups}

As is well known, the nonlinear geometry of Banach spaces and large scale geometry of finitely generated groups share many common concepts and tools, 
while a priori dealing with distinct subject matters. However, as shown in [57], both theories may be viewed as instances of the same overarching framework, namely, the coarse geometry of topological groups. Thus, many results or problems admitting analogous but separate treatments for Banach spaces and groups can in fact be entered into this unified framework.

Recall that, if $G$ is a topological group, the left-uniform structure $\mathcal{U}_{L}$ on $G$ is the uniform structure generated by the family of entourages

$$
E_{V}=\left\{(x, y) \in G \times G \mid x^{-1} y \in V\right\},
$$

where $V$ varies over identity neighbourhoods in $G$. It is a fact due to Weil that the left-uniform structure on $G$ is given as the union $\mathcal{U}_{L}=\bigcup_{d} \mathcal{U}_{d}$ of the uniformities $\mathcal{U}_{d}$ induced by continuous left-invariant écarts (aka. pseudometrics) $d$ on $G$. Thus, $E \in \mathcal{U}_{L}$ if it contains some

$$
E_{\alpha}=\{(x, y) \in X \times X \mid d(x, y)<\alpha\},
$$

with $\alpha>0$ and $d$ a continuous left-invariant écart on $G$. Apart from the weak uniformity on a Banach space, this is the only uniformity on $G$ that we will consider and, in the case of the additive group $(X,+)$ of a Banach space, is simply the uniformity given by the norm.

More recently, in [57] we have developed a theory of coarse geometry of topological groups the basic concepts of which are analogous to those of the left uniformity. For this, we first need Roe's concept of a coarse space [53]. A coarse structure on a set $X$ is a family $\mathcal{E}$ of subsets $E \subseteq X \times X$ called coarse entourages satisfying:

(i) the diagonal $\Delta$ belongs to $\mathcal{E}$;

(ii) $F \subseteq E \in \mathcal{E} \Rightarrow F \in \mathcal{E}$;

(iii) $E \in \mathcal{E} \Rightarrow E^{-1}=\{(y, x) \mid(x, y) \in E\} \in \mathcal{E}$;

(iv) $E, F \in \mathcal{E} \Rightarrow E \cup F \in \mathcal{E}$;

(v) $E, F \in \mathcal{E} \Rightarrow E \circ F=\{(x, y) \mid \exists z(x, z) \in E \&(z, y) \in F\} \in \mathcal{E}$.

Just as the prime example of a uniform space is a metric space, the motivating example of a coarse space $(X, \mathcal{E})$ is that induced from a (pseudo) metric space $(X, d)$. Indeed, in this case, we let $E \in \mathcal{E}_{d}$ if $E$ is contained in some

$$
E_{\alpha}=\{(x, y) \in X \times X \mid d(x, y)<\alpha\}
$$

with $\alpha<\infty$. 
Now, if $G$ is a topological group, we define the left-coarse structure $\mathcal{E}_{L}$ to be the coarse structure given by

$$
\mathcal{E}_{L}=\bigcap_{d} \mathcal{E}_{d}
$$

where the intersection is taken over the family of coarse structures $\mathcal{E}_{d}$ given by continuous left-invariant écarts on $G$. Again, this is the only coarse structure on a topological group we will consider and, in the case of a finitely generated or locally compact, compactly generated group, coincides with the coarse structure given by the (left-invariant) word metric. Similarly, for a Banach space, the coarse structure $\mathcal{E}_{L}$ is simply that given by the norm.

A subset $A$ of a topological group has property $(O B)$ relative to $G$ if $A$ has finite diameter with respect to every continuous left-invariant écart on $G$. Also, $G$ has property $(O B)$ if is has property $(\mathrm{OB})$ relative to itself. A continuous leftinvariant écart $d$ on a topological group $G$ is said to be coarsely proper if it induces the coarse structure, that is, if $\mathcal{E}_{L}=\mathcal{E}_{d}$. In the class of Polish groups, having a coarsely proper écart is equivalent to the group being locally $(O B)$, that is, having a relatively $(\mathrm{OB})$ identity neighbourhood. Finally, $G$ is $(O B)$ generated if it is generated by a relatively $(\mathrm{OB})$ set.

Recall that a map $\sigma:(X, \mathcal{U}) \rightarrow(Y, \mathcal{V})$ between uniform spaces is uniformly continuous if, for every $F \in \mathcal{V}$, there is $E \in \mathcal{U}$ so that $(a, b) \in E \Rightarrow(\sigma(a)$, $\sigma(b)) \in F$. Also, $\sigma$ is a uniform embedding is, moreover, for every $E \in \mathcal{U}$, there is $F \in \mathcal{V}$ so that $(a, b) \notin E \Rightarrow(\sigma(a), \sigma(b)) \notin F$. Similarly, a map $\sigma:(X$, $\mathcal{E}) \rightarrow(Y, \mathcal{F})$ between coarse spaces is bornologous if, for every $E \in \mathcal{E}$, there is $F \in \mathcal{F}$ so that $(a, b) \in E \Rightarrow(\sigma(a), \sigma(b)) \in F$. And $\sigma$ is expanding if, every $F \in \mathcal{F}$, there is $E \in \mathcal{E}$ so that $(a, b) \notin E \Rightarrow(\sigma(a), \sigma(b)) \notin F$. An expanding bornologous map is called a coarse embedding.

A coarse embedding $\sigma$ is a coarse equivalence if, moreover, the image $\sigma[X]$ is cobounded in $Y$, that is, there is some $F \in \mathcal{F}$ so that

$$
\forall y \in Y \exists x \in X(y, \sigma(x)) \in F .
$$

A map $\sigma:(X, \mathcal{E}) \rightarrow(Y, \mathcal{U})$ from a coarse space $X$ to a uniform space $Y$ is uncollapsed if there are a coarse entourage $E \in \mathcal{E}$ and a uniform entourage $F \in \mathcal{U}$ so that

$$
(a, b) \notin E \Rightarrow(\sigma(a), \sigma(b)) \notin F .
$$

These definitions all agree with those given for the specific case of metric spaces.

It turns out to be useful to introduce a finer modulus than the compression. For this, given a map $\sigma:(X, d) \rightarrow(Y, \partial)$ between metric spaces, define the exact compression modulus by

$$
\tilde{\kappa}_{\sigma}(t)=\inf (\partial(\sigma(a), \sigma(b)) \mid d(a, b)=t)
$$

and observe that $\kappa_{\sigma}(t)=\inf _{s \geqslant t} \tilde{\kappa}_{\sigma}(s)$. 


\section{Uniform versus coarse embeddings between Banach spaces}

Observe first that a map $\sigma: X \rightarrow M$ from a Banach space $X$ to a uniform space $(M, \mathcal{U})$ is uncollapsed if there are $\Delta>0$ and an entourage $F \in \mathcal{U}$ so that

$$
\|x-y\|>\Delta \Rightarrow(\sigma(x), \sigma(y)) \notin F .
$$

For example, a uniform embedding is uncollapsed. Note also that, if $\sigma: X \rightarrow G$ is a uniformly continuous uncollapsed map into a topological group $G$, then $x \mapsto(\sigma(x), \sigma(2 x), \sigma(3 x), \ldots)$ defines a uniform embedding of $X$ into the infinite product $\prod_{n \in \mathbb{N}} G$.

Our main results about uncollapsed maps between Banach spaces are as follows.

THEOREM 11. Suppose $\sigma: X \rightarrow E$ is an uncollapsed uniformly continuous map between Banach spaces. Then, for any $1 \leqslant p<\infty, X$ admits a simultaneously uniform and coarse embedding into $\ell^{p}(E)$.

The next corollary then follows from observing that all of the classes of spaces listed are closed under the operation $E \mapsto \ell^{p}(E)$ for an appropriate $1 \leqslant p<\infty$.

COROLlARY 2. If a Banach space $X$ is uniformly embeddable into $\ell^{p}, L^{p}$ (for some $1 \leqslant p<\infty)$, a reflexive, super-reflexive, stable, superstable, nontrivial type or cotype space, then $X$ admits a simultaneously uniform and coarse embedding into a space of the same kind.

If we wish to avoid the passage from $E$ to the infinite sum $\ell^{p}(E)$, we can get by with a direct sum $E \oplus E$, but only assuming that $\sigma$ maps into a bounded set. Also, the resulting map may no longer be a uniform embedding.

THEOREM 12. Suppose $\sigma: X \rightarrow B_{E}$ is an uncollapsed uniformly continuous map from a Banach space $X$ into the ball of a Banach space E. Then $X$ admits a uniformly continuous coarse embedding into $E \oplus E$.

Both propositions will be consequences of somewhat finer and more detailed results with wider applicability. Indeed, Theorem 11 is a direct corollary of Lemma 1 below.

Lemma 1. Suppose $X$ and $E$ are Banach spaces and $P_{n}: E \rightarrow E$ is a sequence of bounded projections onto subspaces $E_{n} \subseteq E$ so that, $E_{m} \subseteq$ ker $P_{n}$ for all $m \neq n$. Assume also that $\sigma_{n}: X \rightarrow E_{n}$ are uncollapsed uniformly continuous maps. Then $X$ admits a simultaneously uniform and coarse embedding into $E$. 
This lemma applies in particular to the case when $E$ is a Schauder sum of a sequence of subspaces $E_{n}$.

Proof. By composing with a translation, we may suppose that $\sigma_{n}(0)=0$ for each $n$. Fix also $\Delta_{n}, \delta_{n}, \epsilon_{n}>0$ so that

$$
\|x-y\| \geqslant \Delta_{n} \Rightarrow\left\|\sigma_{n}(x)-\sigma_{n}(y)\right\| \geqslant \delta_{n}
$$

and

$$
\|x-y\| \leqslant \epsilon_{n} \Rightarrow\left\|\sigma_{n}(x)-\sigma_{n}(y)\right\| \leqslant 2^{-n} .
$$

Note that, if $\|x-y\| \leqslant k \cdot \epsilon_{n}$ for some $k \in \mathbb{N}$, then there are $z_{0}=x, z_{1}, z_{2}, \ldots$, $z_{k}=y \in X$ so that $\left\|z_{i}-z_{i+1}\right\|=(1 / k)\|x-y\| \leqslant \epsilon_{n}$, whence

$$
\left\|\sigma_{n}(x)-\sigma_{n}(y)\right\| \leqslant \sum_{i=1}^{k}\left\|\sigma_{n}\left(z_{i-1}\right)-\sigma_{n}\left(z_{i}\right)\right\| \leqslant k \cdot 2^{-n} .
$$

Thus, setting $\psi_{n}(x)=\sigma_{n}\left(n \Delta_{n} \cdot x\right) /\left\lceil\left(n^{2} \Delta_{n} / \epsilon_{n}\right)\right\rceil$, we have, for all $x, y \in X$,

$$
\begin{aligned}
\|x-y\| \leqslant n & \Rightarrow\left\|n \Delta_{n} \cdot x-n \Delta_{n} \cdot y\right\| \leqslant n^{2} \Delta_{n}<\left\lceil\frac{n^{2} \Delta_{n}}{\epsilon_{n}}\right\rceil \cdot \epsilon_{n} \\
& \Rightarrow\left\|\sigma_{n}\left(n \Delta_{n} \cdot x\right)-\sigma_{n}\left(n \Delta_{n} \cdot y\right)\right\| \leqslant\left\lceil\frac{n^{2} \Delta_{n}}{\epsilon_{n}}\right\rceil \cdot 2^{-n} \\
& \Rightarrow\left\|\psi_{n}(x)-\psi_{n}(y)\right\| \leqslant 2^{-n},
\end{aligned}
$$

while

$$
\begin{aligned}
\|x-y\| \geqslant \frac{1}{n} & \Rightarrow\left\|n \Delta_{n} \cdot x-n \Delta_{n} \cdot y\right\| \geqslant \Delta_{n} \\
& \Rightarrow\left\|\sigma_{n}\left(n \Delta_{n} \cdot x\right)-\sigma_{n}\left(n \Delta_{n} \cdot y\right)\right\| \geqslant \delta_{n} \\
& \Rightarrow\left\|\psi_{n}(x)-\psi_{n}(y)\right\| \geqslant \frac{\delta_{n}}{\left\lceil\left(n^{2} \Delta_{n} / \epsilon_{n}\right)\right\rceil} .
\end{aligned}
$$

Now choose $\xi_{n}>0$ so that

$$
\|x-y\| \leqslant \xi_{n} \Rightarrow\left\|\sigma_{n}(x)-\sigma_{n}(y)\right\| \leqslant \frac{\delta_{n}}{n\left\|P_{n}\right\|} \cdot 2^{-n}
$$

and set $\phi_{n}(x)=\left(n\left\|P_{n}\right\| / \delta_{n}\right) \cdot \sigma_{n}\left(\xi_{n} / n \cdot x\right)$. Then

$$
\begin{aligned}
\|x-y\| \leqslant n & \Rightarrow\left\|\frac{\xi_{n}}{n} \cdot x-\frac{\xi_{n}}{n} \cdot y\right\| \leqslant \xi_{n} \\
& \Rightarrow\left\|\sigma_{n}\left(\frac{\xi_{n}}{n} \cdot x\right)-\sigma_{n}\left(\frac{\xi_{n}}{n} \cdot y\right)\right\| \leqslant \frac{\delta_{n}}{n\left\|P_{n}\right\|} \cdot 2^{-n} \\
& \Rightarrow\left\|\phi_{n}(x)-\phi_{n}(y)\right\| \leqslant 2^{-n},
\end{aligned}
$$


while

$$
\begin{aligned}
\|x-y\| \geqslant \frac{n \Delta_{n}}{\xi_{n}} & \Rightarrow\left\|\frac{\xi_{n}}{n} \cdot x-\frac{\xi_{n}}{n} \cdot y\right\| \geqslant \Delta_{n} \\
& \Rightarrow\left\|\sigma_{n}\left(\frac{\xi_{n}}{n} \cdot x\right)-\sigma_{n}\left(\frac{\xi_{n}}{n} \cdot y\right)\right\| \geqslant \delta_{n} \\
& \Rightarrow\left\|\phi_{n}(x)-\phi_{n}(y)\right\| \geqslant n\left\|P_{n}\right\| .
\end{aligned}
$$

In particular, if $\|x-y\| \leqslant m$, then $\|x-y\| \leqslant n$ for all $n \geqslant m$, whence

$$
\sum_{n=1}^{\infty}\left\|\psi_{2 n-1}(x)-\psi_{2 n-1}(y)\right\| \leqslant \sum_{n=1}^{m-1}\left\|\psi_{2 n-1}(x)-\psi_{2 n-1}(y)\right\|+\sum_{n=m}^{\infty} 2^{-2 n+1}<\infty
$$

and

$$
\sum_{n=1}^{\infty}\left\|\phi_{2 n}(x)-\phi_{2 n}(y)\right\| \leqslant \sum_{n=1}^{m-1}\left\|\phi_{2 n}(x)-\phi_{2 n}(y)\right\|+\sum_{n=m}^{\infty} 2^{-2 n}<\infty .
$$

Setting $y=0$, we see that both $\sum_{n=1}^{\infty} \psi_{2 n-1}(x)$ and $\sum_{n=1}^{\infty} \phi_{2 n}(x)$ are absolutely convergent in $E$, whence we may define $\omega: X \rightarrow E$ by

$$
\omega(x)=\sum_{n=1}^{\infty} \psi_{2 n-1}(x)+\sum_{n=1}^{\infty} \phi_{2 n}(x) .
$$

First, to see that $\omega$ is uniformly continuous and thus bornologous, let $\epsilon>0$ and find $m$ large enough so that $2^{-2 m+2}<\epsilon / 3$. Since each of $\sigma_{n}$ is uniformly continuous, so are the $\psi_{n}$ and $\phi_{n}$. We may therefore choose $\eta>0$ so that

$$
\left\|\left(\sum_{n=1}^{m-1} \psi_{2 n-1}(x)+\sum_{n=1}^{m-1} \phi_{2 n}(x)\right)-\left(\sum_{n=1}^{m-1} \psi_{2 n-1}(y)+\sum_{n=1}^{m-1} \phi_{2 n}(y)\right)\right\|<\frac{\epsilon}{3}
$$

whenever $\|x-y\|<\eta$. Thus, if $\|x-y\|<\min \{\eta, m\}$, we have

$$
\begin{aligned}
\|\omega(x)-\omega(y)\| \leqslant & \|\left(\sum_{n=1}^{m-1} \psi_{2 n-1}(x)+\sum_{n=1}^{m-1} \phi_{2 n}(x)\right) \\
& -\left(\sum_{n=1}^{m-1} \psi_{2 n-1}(y)+\sum_{n=1}^{m-1} \phi_{2 n}(y)\right) \| \\
& +\sum_{n=m}^{\infty}\left\|\psi_{2 n-1}(x)-\psi_{2 n-1}(y)\right\|+\sum_{n=m}^{\infty}\left\|\phi_{2 n}(x)-\phi_{2 n}(y)\right\| \\
< & \frac{\epsilon}{3}+\frac{\epsilon}{3}+\frac{\epsilon}{3},
\end{aligned}
$$

showing uniform continuity. 
Secondly, to see that $\omega$ is a uniform embedding, suppose that $\|x-y\|>$ $1 /(2 n-1)$ for some $n \geqslant 1$. Then,

$$
\begin{aligned}
\|\omega(x)-\omega(y)\| & \geqslant \frac{1}{\left\|P_{2 n-1}\right\|}\left\|P_{2 n-1} \omega(x)-P_{2 n-1} \omega(y)\right\| \\
& \geqslant \frac{1}{\left\|P_{2 n-1}\right\|}\left\|\psi_{2 n-1}(x)-\psi_{2 n-1}(y)\right\| \\
& \geqslant \frac{1}{\left\|P_{2 n-1}\right\|} \cdot \frac{\delta_{2 n-1}}{\left\lceil(2 n-1)^{2} \Delta_{2 n-1} / \epsilon_{2 n-1}\right\rceil} .
\end{aligned}
$$

Finally, to see that $\omega$ is a coarse embedding, observe that, if $\|x-y\| \geqslant$ $\left(2 n \Delta_{2 n} / \xi_{2 n}\right)$, then

$$
\begin{aligned}
\|\omega(x)-\omega(y)\| & \geqslant \frac{1}{\left\|P_{2 n}\right\|}\left\|P_{2 n} \omega(x)-P_{2 n} \omega(y)\right\| \\
& \geqslant \frac{1}{\left\|P_{2 n}\right\|}\left\|\phi_{2 n}(x)-\phi_{2 n}(y)\right\| \\
& \geqslant 2 n,
\end{aligned}
$$

which finishes the proof.

We should mention here that Braga [13] has been able to use our construction above coupled with a result of Odell and Schlumprecht [48] to show that $\ell^{2}$ admits a simultaneously uniform and coarse embedding into every Banach space with an unconditional basis and finite cotype.

Our next result immediately implies theorem 12.

Lemma 2. Suppose $\sigma: X \rightarrow B_{E}$ and $\omega: X \rightarrow B_{F}$ are uncollapsed uniformly continuous maps from a Banach space $X$ into the balls of Banach spaces $E$ and $F$. Then $X$ admits uniformly continuous coarse embedding into $E \oplus F$.

Proof. Since $\sigma$ and $\omega$ are uncollapsed, pick $\Delta \geqslant 2$ and $\delta>0$ so that

$$
\|x-y\|>\Delta \Rightarrow\|\sigma(x)-\sigma(y)\|>\delta \quad \& \quad\|\omega(x)-\omega(y)\|>\delta .
$$

We will inductively define bounded uniformly continuous maps

$$
\phi_{1}, \phi_{2}, \ldots: X \rightarrow E
$$

and

$$
\psi_{1}, \psi_{2}, \ldots: Y \rightarrow F
$$


with $\phi_{n}(0)=\psi_{n}(0)=0$ and numbers $0=t_{0}<r_{1}<t_{1}<r_{2}<t_{2}<\cdots$ with $\lim _{n} r_{n}=\infty$ so that, for all $n \geqslant 1$,

$$
\begin{gathered}
\|x-y\| \geqslant r_{n} \Rightarrow\left\|\sum_{i=1}^{n} \phi_{i}(x)-\sum_{i=1}^{n} \phi_{i}(y)\right\| \geqslant 2^{n}, \\
\|x-y\| \geqslant t_{n} \Rightarrow\left\|\sum_{i=1}^{n} \psi_{i}(x)-\sum_{i=1}^{n} \psi_{i}(y)\right\| \geqslant 2^{n}, \\
\|x-y\| \leqslant t_{n-1} \Rightarrow\left\|\phi_{n}(x)-\phi_{n}(y)\right\| \leqslant 2^{-n}
\end{gathered}
$$

and

$$
\|x-y\| \leqslant r_{n} \Rightarrow\left\|\psi_{n}(x)-\psi_{n}(y)\right\| \leqslant 2^{-n} .
$$

Suppose that this have been done. Then

$$
\|x-y\| \leqslant t_{n-1} \Rightarrow \sum_{i=n}^{\infty}\left\|\phi_{i}(x)-\phi_{i}(y)\right\| \leqslant \sum_{i=n}^{\infty} 2^{-i} \leqslant 1 .
$$

In particular, setting $y=0$, we see that the series $\sum_{i=1}^{\infty} \phi_{i}(x)$ is absolutely convergent for all $x \in X$. Similarly,

$$
\|x-y\| \leqslant r_{n} \Rightarrow \sum_{i=n}^{\infty}\left\|\psi_{i}(x)-\psi_{i}(y)\right\| \leqslant \sum_{i=n}^{\infty} 2^{-i} \leqslant 1,
$$

showing that also $\sum_{i=1}^{\infty} \psi_{i}(x)$ is absolutely convergent for all $x \in X$. So define $\phi: X \rightarrow E$ and $\psi: X \rightarrow F$ by $\phi(x)=\sum_{i=1}^{\infty} \phi_{i}(x)$ and $\psi(x)=\sum_{i=1}^{\infty} \psi_{i}(x)$.

We claim that $\phi$ and $\psi$ are uniformly continuous. To see this, let $\alpha>0$ be given and pick $n \geqslant 2$ so that $2^{-n+2}<\alpha$. By uniform continuity of the $\phi_{i}$, we may choose $\beta>0$ small enough so that $\sum_{i=1}^{n-1}\left\|\phi_{i}(x)-\phi_{i}(y)\right\|<\alpha / 2$ whenever $\|x-y\|<\beta$. Thus, if $\|x-y\|<\min \left\{\beta, t_{n-1}\right\}$, we have

$$
\begin{aligned}
\|\phi(x)-\phi(y)\| & \leqslant \sum_{i=1}^{n-1}\left\|\phi_{i}(x)-\phi_{i}(y)\right\|+\sum_{i=n}^{\infty}\left\|\phi_{i}(x)-\phi_{i}(y)\right\| \\
& <\frac{\alpha}{2}+\sum_{i=n}^{\infty} 2^{-i} \\
& <\alpha,
\end{aligned}
$$

showing that $\phi$ is uniformly continuous. A similar argument works for $\psi$. 
Now, suppose $\|x-y\| \geqslant r_{m}$. Then either $r_{n} \leqslant\|x-y\| \leqslant t_{n}$ or $t_{n} \leqslant\|x-y\| \leqslant$ $r_{n+1}$ for some $n \geqslant m$. In the first case,

$$
\begin{aligned}
\|\phi(x)-\phi(y)\| & =\left\|\sum_{i=1}^{\infty} \phi_{i}(x)-\sum_{i=1}^{\infty} \phi_{i}(y)\right\| \\
& \geqslant\left\|\sum_{i=1}^{n} \phi_{i}(x)-\sum_{i=1}^{n} \phi_{i}(y)\right\|-\sum_{i=n+1}^{\infty}\left\|\phi_{i}(x)-\phi_{i}(y)\right\| \\
& \geqslant 2^{n}-1,
\end{aligned}
$$

while, in the second case,

$$
\begin{aligned}
\|\psi(x)-\psi(y)\| & =\left\|\sum_{i=1}^{\infty} \psi_{i}(x)-\sum_{i=1}^{\infty} \psi_{i}(y)\right\| \\
& \geqslant\left\|\sum_{i=1}^{n} \psi_{i}(x)-\sum_{i=1}^{n} \psi_{i}(y)\right\|-\sum_{i=n+1}^{\infty}\left\|\psi_{i}(x)-\psi_{i}(y)\right\| \\
& \geqslant 2^{n}-1 .
\end{aligned}
$$

Thus,

$$
\|x-y\| \geqslant r_{m} \Rightarrow\|\phi(x)-\phi(y)\|+\|\psi(x)-\psi(y)\| \geqslant 2^{m}-1,
$$

showing that $\phi \oplus \psi: X \rightarrow E \oplus F$ is expanding. As each of $\phi$ and $\psi$ is uniformly continuous, so is $\phi \oplus \psi$ and therefore also bornologous. It follows that $\phi \oplus \psi$ is a coarse embedding of $X$ into $E \oplus F$.

Let us now return to the construction of $\phi_{i}, \psi_{i}, r_{i}$ and $t_{i}$. We begin by letting $t_{0}=0, r_{1}=\Delta$ and $\phi_{1}=(2 / \delta) \sigma$. Now suppose that $\phi_{1}, \ldots, \phi_{n}, \psi_{1}, \ldots, \psi_{n-1}$ and $t_{0}<r_{1}<t_{1}<\cdots<r_{n}$ have been defined satisfying the required conditions. As the $\psi_{i}$ are bounded, let

$$
S=\sup _{x, y \in X}\left\|\sum_{i=1}^{n-1} \psi_{i}(x)-\sum_{i=1}^{n-1} \psi_{i}(y)\right\| .
$$

Also, as $\omega$ is uniformly continuous, pick $0<\epsilon<1$ so that $\|\omega(x)-\omega(y)\|<$ $\delta /\left(S+2^{n}\right) 2^{n}$ whenever $\|x-y\| \leqslant \epsilon$ and let $\psi_{n}(x)=\left(\left(S+2^{n}\right) / \delta\right) \omega\left(\left(\epsilon / r_{n}\right) x\right)$. Then, if $\|x-y\| \leqslant r_{n}$, we have $\left\|\left(\epsilon / r_{n}\right) x-\left(\epsilon / r_{n}\right) y\right\| \leqslant \epsilon$ and so

$$
\left\|\psi_{n}(x)-\psi_{n}(y)\right\|=\frac{S+2^{n}}{\delta}\left\|\omega\left(\frac{\epsilon}{r_{n}} x\right)-\omega\left(\frac{\epsilon}{r_{n}} y\right)\right\|<2^{-n} .
$$


On the other hand, if we let $t_{n}=r_{n} \Delta / \epsilon$, then

$$
\|x-y\| \geqslant t_{n} \Rightarrow\left\|\psi_{n}(x)-\psi_{n}(y)\right\| \geqslant S+2^{n} \Rightarrow\left\|\sum_{i=1}^{n} \psi_{i}(x)-\sum_{i=1}^{n} \psi_{i}(y)\right\| \geqslant 2^{n} .
$$

Note that, as $\epsilon<1$ and $\Delta \geqslant 2$, we have $t_{n}>2 r_{n}$. A similar construction allows us to find $\phi_{n+1}$ and $r_{n+1}>2 t_{n}$ given $\phi_{1}, \ldots, \phi_{n}, \psi_{1}, \ldots, \psi_{n}$ and $t_{0}<r_{1}$ $<\cdots<t_{n}$.

In the light of the previous results, it would be very interesting to determine when a coarse embedding can be replaced by a uniformly continuous coarse embedding. As mentioned earlier, Naor [46] was able to construct a bornologous map between two separable Banach spaces, which is not close to any uniformly continuous map.

QUESTION 2. Suppose X is a separable Banach space coarsely embedding into a separable Banach space E. Is there a uniformly continuous coarse embedding of $X$ into $E$ ?

\section{Cocycles and affine isometric representations}

By the Mazur-Ulam Theorem, every surjective isometry $A$ of a Banach space $X$ is affine, that is, there are a unique invertible linear isometry $T: X \rightarrow X$ and a vector $\eta \in X$ so that $A$ is given by $A(\xi)=T(\xi)+\eta$ for all $\xi \in X$. It follows that, if $\alpha: G \curvearrowright X$ is an isometric action of a group $G$ on a Banach space $X$, there is an isometric linear representation $\pi: G \curvearrowright X$, called the linear part of $\alpha$, and a corresponding cocycle $b: G \rightarrow X$ so that

$$
\alpha(g) \xi=\pi(g) \xi+b(g)
$$

for all $g \in G$ and $\xi \in X$. In particular, $b$ is simply the orbit map $g \mapsto \alpha(g) 0$. Moreover, the cocycle $b$ then satisfies the cocycle equation

$$
b(g f)=\pi(g) b(f)+b(g)
$$

for $g, f \in G$. Finally, as $\alpha$ is an action by isometries, we have

$$
\begin{aligned}
\|b(f)-b(g)\| & =\|\alpha(f) 0-\alpha(g) 0\| \\
& =\left\|\alpha\left(g^{-1} f\right) 0-0\right\| \\
& =\left\|b\left(g^{-1} f\right)\right\| .
\end{aligned}
$$

Now, if $G$ is a topological group, the action $\alpha$ is continuous, that is, continuous as a map $\alpha: G \times X \rightarrow X$, if and only if the linear part $\pi$ is strongly continuous, 
that is, $g \in G \mapsto \pi(g) \xi \in X$ is continuous for every $\xi \in X$, and $b: G \rightarrow X$ is continuous. Moreover, since $b$ is simply the orbit map $g \mapsto \alpha(g) 0$, in this case, the cocycle $b: G \rightarrow X$ is both uniformly continuous and bornologous. We say that the action $\alpha$ is coarsely proper if and only if $b: G \rightarrow X$ is a coarse embedding.

If $\pi: G \curvearrowright X$ is a strongly continuous isometric linear representation, we let $Z^{1}(G, \pi)$ denote the vector space of continuous cocycles $b: G \rightarrow X$ associated to $\pi$. Also, let $B^{1}(G, \pi)$ denote the linear subspace of coboundaries, that is, cocycles $b$ of the form $b(g)=\xi-\pi(g) \xi$ for some $\xi \in X$. Note that the cocycle $b$ has this form if and only if $\xi$ is fixed by the corresponding affine isometric action $\alpha$ induced by $\pi$ and $b$.

As noted above, continuous cocycles are actually uniformly continuous. But, in the case of cocycles between Banach spaces, we have stronger information available.

Proposition 1. Let $b: X \rightarrow E$ be a continuous uncollapsed cocycle between Banach spaces $X$ and $E$, that is, there are $\Delta, \delta>0$ so that

$$
\|x\|>\Delta \Rightarrow\|b(x)\|>\delta .
$$

Then $b: X \rightarrow E$ is a uniform embedding. In fact, there are constants $c, C>0$ so that

$$
c \cdot \min \{\|x-y\|, 1\} \leqslant\|b(x)-b(y)\| \leqslant C\|x-y\|+C .
$$

Proof. Suppose that $\pi: X \curvearrowright E$ is the strongly continuous isometric linear action for which $b: X \rightarrow E$ an uncollapsed continuous cocycle. As noted above, $b$ is uniformly continuous and hence, by the Corson-Klee lemma [11, Proposition 1.11], also Lipschitz for large distances. This shows the second inequality.

We now show the first inequality with $c=\min \{\delta, \delta / 2 \Delta\}$, which implies uniform continuity of $b^{-1}$. Since $\|b(x)-b(y)\|=\|b(x-y)\|$, it suffices to verify that

$$
c \cdot \min \{\|x\|, 1\} \leqslant\|b(x)\|
$$

for all $x \in X$. For $\|x\|>\Delta$, this follows from our assumption and choice of $c$. So suppose instead that $x \in X \backslash\{0\}$ with $\|x\| \leqslant \Delta$ and let $n$ be minimal so that $n\|x\|>\Delta$. Then $\|x\| \leqslant \Delta /(n-1) \leqslant 2 \Delta / n$ and

$$
\begin{aligned}
\delta & <\|b(n \cdot x)\| \\
& =\left\|\pi^{n-1}(x) b(x)+\pi^{n-2}(x) b(x)+\cdots+b(x)\right\| \\
& \leqslant\left\|\pi^{n-1}(x) b(x)\right\|+\left\|\pi^{n-2}(x) b(x)\right\|+\cdots+\|b(x)\| \\
& =n \cdot\|b(x)\|,
\end{aligned}
$$


that is,

$$
\|b(x)\| \geqslant \frac{\delta}{n} \geqslant \frac{\delta}{2 \Delta} \cdot \frac{2 \Delta}{n} \geqslant \frac{\delta}{2 \Delta} \cdot\|x\| \geqslant c\|x\|
$$

as required.

Since any continuous cocycle is both bornologous and uniformly continuous, we see that any coarsely proper continuous cocycle $b: X \rightarrow E$ between Banach spaces is simultaneously a uniform and coarse embedding.

The following variation is also of independent interest.

Proposition 2. Let $b: X \rightarrow E$ be a continuous cocycle between Banach spaces satisfying

$$
\inf _{\|x\|=r}\|b(x)\|>0
$$

for some $r>0$. Then $B_{X}$ uniformly embeds into $B_{E}$.

Proof. Set $\delta=\inf _{\|x\|=r}\|b(x)\|$ and find, by uniform continuity of $b$, some $m$ so that

$$
\|b(x)\|>\frac{\delta}{2}
$$

whenever $r \leqslant\|x\| \leqslant r+2 / m$. Then, if $0<\|x\| \leqslant 1 / m$, fix $n$ minimal so that $r \leqslant\|n x\| \leqslant r+2 / m$. With this choice of $n$ we have as in the proof of Proposition 1 that

$$
\|b(x)\| \geqslant \frac{\delta}{2 n} \geqslant \frac{\delta}{4 r}\|x\|,
$$

showing that $b:(1 / m) B_{X} \rightarrow E$ is a uniform embedding. The proposition follows by rescaling $b$.

Though we shall return to the issue later, let us just mention that uniform embeddings between balls of Banach spaces has received substantial attention. For example, Raynaud [52] (see also [11, Section 9.5]) has shown that $B_{c_{0}}$ does not uniformly embed into a stable metric space, for example, into $L^{p}([0,1])$ with $1 \leqslant p<\infty$.

Our next result replicates the construction from Section 3 within the context of cocycles.

Proposition 3. Suppose $b: X \rightarrow E$ is an uncollapsed continuous cocycle between Banach spaces. Then, for every $1 \leqslant p<\infty$, there is a coarsely proper continuous cocycle $\tilde{b}: X \curvearrowright \ell^{p}(E)$. 
Proof. Let $b$ be associated to the strongly continuous isometric linear representation $\pi: X \curvearrowright E$. As in the proof of Lemma 11, there are constants $\epsilon_{n}$ and $K_{n}$ so that

$$
x \mapsto\left(K_{1} \cdot b\left(\epsilon_{1} x\right), K_{2} \cdot b\left(\epsilon_{2} x\right), \ldots\right)
$$

defines a simultaneously uniform and coarse embedding of $X$ into $\ell^{p}(E)$. We claim that the map $\tilde{b}: X \rightarrow \ell^{p}(E)$ so defined is a cocycle for some strongly continuous isometric linear representation $\tilde{\pi}: X \curvearrowright \ell^{p}(E)$. Indeed, observe that each $x \mapsto K_{n} \cdot b\left(\epsilon_{n} x\right)$ is a cocycle for the isometric linear representation $x \mapsto$ $\pi\left(\epsilon_{n} x\right)$ on $E$, so $\tilde{b}$ is a cocycle for the isometric linear representation

$$
\tilde{\pi}(x)=\pi\left(\epsilon_{1} x\right) \otimes \pi\left(\epsilon_{2} x\right) \otimes \cdots
$$

of $X$ on $\ell^{p}(E)$.

LEMMA 3. Let E be a separable Banach space and $\operatorname{Isom}(E)$ the group of linear isometries of $E$ equipped with the strong operator topology. If $\pi: \operatorname{Isom}(E) \curvearrowright$ $\ell^{p}(E)$ denotes the diagonal isometric linear representation for $1 \leqslant p<\infty$, there is a continuous cocycle $b: \operatorname{Isom}(E) \rightarrow \ell^{p}(E)$ associated to $\pi$ that is a uniform embedding of $\operatorname{Isom}(E)$ into $\ell^{p}(E)$.

Proof. Fix a dense subset $\left\{\xi_{n}\right\}_{n \in \mathbb{N}}$ of the sphere $S_{E}$ and let

$$
b(g)=\left(\frac{\xi_{1}-g\left(\xi_{1}\right)}{2^{1}}, \frac{\xi_{2}-g\left(\xi_{2}\right)}{2^{2}}, \frac{\xi_{3}-g\left(\xi_{3}\right)}{2^{3}}, \ldots\right) .
$$

Then $b$ is easily seen to be a cocycle for $\pi$. Moreover, by the definition of the strong operator topology, $b$ is a uniform embedding.

Proposition 4. Suppose $\pi: X \rightarrow \operatorname{Isom}(E)$ is an uncollapsed strongly continuous isometric linear representation of a Banach space $X$ on a separable Banach space $E$. Then, for every $1 \leqslant p<\infty, X$ admits coarsely proper continuous cocycle $b: X \rightarrow \ell^{p}(E)$.

Proof. Let $c: \operatorname{Isom}(E) \rightarrow \ell^{p}(E)$ be the cocycle given by Lemma 3 associated to the diagonal isometric linear representation $\rho: \operatorname{Isom}(E) \curvearrowright \ell^{p}(E)$. It follows that $c \circ \pi: X \rightarrow \ell^{p}(E)$ is a continuous uncollapsed cocycle associated to the isometric linear representation $\rho \circ \pi: X \curvearrowright \ell^{p}(E)$. By Proposition 3 and the fact that $\ell^{p}\left(\ell^{p}(E)\right)=\ell^{p}(E)$, we obtain a coarsely proper continuous cocycle $b: X \rightarrow \ell^{p}(E)$.

Cocycles between Banach spaces are significantly more structured maps than general maps. For example, as shown in Proposition 1, a coarsely proper 
continuous cocycle $b: X \rightarrow E$ between Banach spaces $X$ and $E$ is automatically both a uniform and coarse embedding, but, moreover, $b$ also preserves a certain amount of algebraic structure, depending on the isometric linear representation $\pi: X \curvearrowright E$ of which it is a cocycle.

To study these algebraic features, we must introduce a topology on the space of cocycles. So fix a strongly continuous isometric linear representation $\pi: G \curvearrowright E$ of a topological group $G$ on a Banach space $E$. Every compact set $K \subseteq G$ determines a seminorm $\|\cdot\|_{K}$ on $Z^{1}(G, \pi)$ by $\|b\|_{K}=\sup _{g \in K}\|b(g)\|$ and the family of seminorms thus obtained endows $Z^{1}(G, \pi)$ with a locally convex topology. With this topology, one sees that a cocycle $b$ belongs to the closure $\overline{B^{1}(G, \pi)}$ if and only if the corresponding affine action $\alpha=(\pi, b)$ almost has fixed points, that is, if for any compact set $K \subseteq G$ and $\epsilon>0$ there is some $\xi=\xi_{K, \epsilon} \in E$ verifying

$$
\sup _{x \in K}\|(\pi(x) \xi+b(x))-\xi\|=\sup _{x \in K}\|b(x)-(\xi-\pi(x) \xi)\|<\epsilon .
$$

Elements of $\overline{B^{1}(G, \pi)}$ are called almost coboundaries.

Note that, if $b$ is a coboundary, then $b(G)$ is a bounded subset of $E$. Conversely, suppose $b(G)$ is a bounded set and $E$ is reflexive. Then any orbit $\mathcal{O}$ of the corresponding affine action is bounded and its closed convex hull $C=\overline{\operatorname{conv}}(\mathcal{O})$ is a weakly compact convex set on which $G$ acts by affine isometries. It follows by the Ryll-Nardzewski fixed point theorem [58] that $G$ fixes a point on $C$, meaning that $b$ must be a coboundary.

Now, if $b \in \overline{B^{1}(G, \pi)}$ and, for every compact $K \subseteq G$, we can choose $\xi=\xi_{K, 1}$ above to have arbitrarily large norm, we see that the supremum

$$
\sup _{x \in K}\left\|\pi(x) \frac{\xi}{\|\xi\|}-\frac{\xi}{\|\xi\|}\right\|<\frac{\sup _{x \in K}\|b(x)\|+1}{\|\xi\|}
$$

can be made arbitrarily small, which means that the linear action $\pi$ almost has invariant unit vectors. If, on the other hand, for some $K$ the choice of $\xi_{K, 1}$ is bounded (but nonempty), then the same bound holds for any compact $K^{\prime} \supseteq K$, whereby we find that $b(G) \subseteq E$ is a bounded set and so, assuming $E$ is reflexive, that $b \in B^{1}(G, \pi)$. This shows that, if $E$ is reflexive and $\pi$ does not almost have invariant unit vectors, then $B^{1}(G, \pi)$ is closed in $Z^{1}(G, \pi)$.

We define the first cohomology group of $G$ with coefficients in $\pi$ to be the quotient space $H^{1}(G, \pi)=Z^{1}(G, \pi) / B^{1}(G, \pi)$, while the reduced cohomology group is $\overline{H^{1}}(G, \pi)=Z^{1}(G, \pi) / \overline{B^{1}(G, \pi)}$.

If $E$ is separable and reflexive, the Alaoglu-Birkhoff decomposition theorem [4] implies that $E$ admits $\pi(G)$-invariant decomposition into closed linear subspaces $E=E^{G} \oplus E_{G}$, where $E^{G}$ is the set of $\pi(G)$-fixed vectors. 
We can therefore write $b=b^{G} \oplus b_{G}$, where $b^{G}: X \rightarrow E^{G}$ and $b_{G}: X \rightarrow E_{G}$ are cocycles for $\pi$. In particular,

$$
b^{G}(x y)=\pi(x) b^{G}(y)+b^{G}(x)=b^{G}(y)+b^{G}(x)=b^{G}(x)+b^{G}(y),
$$

that is, $b^{G}$ is a continuous homomorphism from $G$ to $\left(E^{G},+\right)$. Also, [7, Theorem 2], implies that, if $G$ is abelian, then $\overline{H^{1}}\left(G,\left.\pi\right|_{E_{G}}\right)=0$ and so $b_{G} \in$

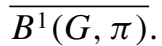

Now, suppose $\pi: X \curvearrowright E$ is a strongly continuous isometric linear representation of a separable Banach space $X$ on a separable reflexive Banach space $E$. Assume that $b: X \rightarrow E$ is a continuous cocycle and let $E=E^{X} \oplus E_{X}$ and $b^{X}: X \rightarrow E^{X}$ and $b_{X}: X \rightarrow E_{X}$ be the decompositions as above. Being a continuous additive homomorphism, $b^{X}$ is a bounded linear operator from $X$ to $E^{X}$. Also, if $b^{X}$ is coarsely proper or even just uncollapsed, then $b^{X}$ must be an isomorphic embedding of $X$ into $E^{X}$.

Now, since $X$ is abelian, $b_{X}: X \rightarrow E_{X}$ belongs to $\overline{B^{1}(X, \pi)}$, which means that, for every norm-compact subset $C \subseteq X$ and $\epsilon>0$, there is $\xi \in E_{X}$ so that

$$
\left\|\xi-\pi(x) \xi-b_{X}(x)\right\|<\epsilon
$$

for all $x \in C$.

We summarize the discussion so far in the following lemma.

Lemma 4. Suppose $\pi: X \curvearrowright E$ is a strongly continuous isometric linear representation of a separable Banach space $X$ on a separable reflexive Banach space $E$ and assume that $b: X \rightarrow E$ is a continuous cocycle. Then there is a $\pi(X)$-invariant decomposition $E=E^{X} \oplus E_{X}$ and a decomposition $b=b^{X} \oplus b_{X}$ so that $b^{X}: X \rightarrow E^{X}$ is a bounded linear operator and $b_{X}: X \rightarrow E_{X}$ belongs to

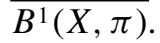

\section{Amenability}

Central in our investigation is the concept of amenability, which for general topological groups is defined as follows.

DEFINITION 1. A topological group $G$ is amenable if every continuous action $\alpha: G \curvearrowright C$ by affine homeomorphisms on a compact convex subset $C$ of a locally convex topological vector space $V$ has a fixed point in $C$.

If $G$ is a topological group, we let $\operatorname{LUC}(G)$ be the vector space of bounded left-uniformly continuous functions $\phi: G \rightarrow \mathbb{R}$ and equip it with the supremum norm induced from the inclusion $\operatorname{LUC}(G) \subseteq \ell^{\infty}(G)$. It then follows that the 
right-regular representation $\rho: G \curvearrowright \operatorname{LUC}(G), \rho(g)(\phi)=\phi(\cdot g)$, is continuous. Moreover, if $G$ is amenable, there exists a $\rho$-invariant mean, that is, a positive continuous linear functional $\mathfrak{m}: \operatorname{LUC}(G) \rightarrow \mathbb{R}$ with $\mathfrak{m}(\mathbf{1})=1$, where $\mathbf{1}$ is the function with constant value 1 , and so that $\mathfrak{m}(\rho(g)(\phi))=\mathfrak{m}(\phi)$.

While we shall use the existence of invariant means on $\operatorname{LUC}(G)$, at one point this does not seem to suffice. Instead, we shall rely on an appropriate generalization of Følner sets present under additional assumptions.

DEFINITION 2. A topological group $G$ is said to be approximately compact if there is a countable chain $K_{0} \leqslant K_{1} \leqslant \cdots \leqslant G$ of compact subgroups whose union $\bigcup_{n} K_{n}$ is dense in $G$.

This turns out to be a fairly common phenomenon among nonlocally compact Polish groups. For example, the unitary group $U(\mathcal{H})$ of separable infinitedimensional Hilbert space with the strong operator topology is approximately compact. Indeed, if $\mathcal{H}_{1} \subseteq \mathcal{H}_{2} \subseteq \cdots \subseteq \mathcal{H}$ is an increasing exhaustive sequence of finite-dimensional subspaces and $U(n)$ denotes the group of unitaries pointwise fixing the orthogonal complement $\mathcal{H}_{n}^{\perp}$, then each $U(n)$ is compact and the union $\bigcup_{n} U(n)$ is dense in $U(\mathcal{H})$.

More generally, as shown by de la Harpe [31], if $M$ is an approximately finite-dimensional von Neumann algebra, that is, there is an increasing sequence $A_{1} \subseteq A_{2} \subseteq \cdots \subseteq M$ of finite-dimensional matrix algebras whose union is dense in $M$ with respect the strong operator topology, then the unitary subgroup $U(M)$ is approximately compact with respect to the strong operator topology.

Similarly, if $G$ contains a locally finite dense subgroup, this will witness approximate compactness. Again this applies to, for example, Aut $([0,1], \lambda)$ with the weak topology, where the dyadic permutations are dense, and $\operatorname{Isom}(\mathbb{U})$ with the pointwise convergence topology (this even holds for the dense subgroup $\operatorname{Isom}(\mathbb{Q U})$ by an unpublished result of Solecki; see [56] for a proof).

Of particular interest to us is the case of non-Archimedean Polish groups. By general techniques, these may be represented as automorphism groups of countable locally finite (i.e., any finitely generated substructure is finite) ultrahomogeneous structures. And, in this setting, we have the following reformulation of approximate compactness.

Proposition 5 (Kechris \& Rosendal [37]). Let $\mathbf{M}$ be a locally finite, countable, ultrahomogeneous structure. Then $\operatorname{Aut}(\mathbf{M})$ is approximately compact if and only if, for every finite substructure $\mathbf{A} \subseteq \mathbf{M}$ and all partial automorphisms $\phi_{1}, \ldots, \phi_{n}$ of $\mathbf{A}$, there is a finite substructure $\mathbf{A} \subseteq \mathbf{B} \subseteq \mathbf{M}$ and full automorphisms $\psi_{1}, \ldots$, $\psi_{n}$ of $\mathbf{B}$ extending $\phi_{1}, \ldots, \phi_{n}$ respectively. 
Whereas a locally compact group is amenable if and only if it admits a Følner sequence, there is no similar characterization of general amenable groups. Nevertheless, one may sometimes get by with a little less, which we isolate in the following definition.

Definition 3. A Polish group $G$ is said to be Følner amenable if either:

(1) $G$ is approximately compact; or

(2) there is a continuous homomorphism $\phi: H \rightarrow G$ from a locally compact second countable amenable group $H$ so that $G=\overline{\phi[H]}$.

Apart from the approximately compact or locally compact amenable groups, easy examples of Følner amenable Polish groups are, for example, Banach spaces or, more generally, abelian groups. Indeed, every abelian Polish group $G$ contains a countable dense subgroup $\Gamma$, which, viewed as a discrete group, is amenable and maps densely into $G$.

\section{Embeddability in Hilbert spaces}

We shall now consider Hilbert valued cocycles, for which we need some background material on kernels conditionally of negative type. The well-known construction of inner products presented here originates in work of Moore [45]. A full treatment can be found, for example, in [9, Appendix C].

DEFINITION 4. A (real-valued) kernel conditionally of negative type on a set $X$ is a function $\Psi: X \times X \rightarrow \mathbb{R}$ so that:

(1) $\Psi(x, x)=0$ and $\Psi(x, y)=\Psi(y, x)$ for all $x, y \in X$;

(2) for all $x_{1}, \ldots, x_{n} \in X$ and $r_{1}, \ldots, r_{n} \in \mathbb{R}$ with $\sum_{i=1}^{n} r_{i}=0$; we have

$$
\sum_{i=1}^{n} \sum_{j=1}^{n} r_{i} r_{j} \Psi\left(x_{i}, x_{j}\right) \leqslant 0
$$

For example, if $\sigma: X \rightarrow \mathcal{H}$ is any mapping from $X$ into a Hilbert space $\mathcal{H}$, then a simple calculation shows that

$$
\sum_{i=1}^{n} \sum_{j=1}^{n} r_{i} r_{j}\left\|\sigma\left(x_{i}\right)-\sigma\left(x_{j}\right)\right\|^{2}=-2\left\|\sum_{i=1}^{n} r_{i} \sigma\left(x_{i}\right)\right\| \leqslant 0,
$$


whenever $\sum_{i=1}^{n} r_{i}=0$, which implies that $\Psi(x, y)=\|\sigma(x)-\sigma(y)\|^{2}$ is a kernel conditionally of negative type.

Suppose that $\Psi$ is a kernel conditionally of negative type on a set $X$ and let $\mathbb{M}(X)$ denote the vector space of finitely supported real-valued functions $\xi$ on $X$ of mean 0 , that is, $\sum_{x \in X} \xi(x)=0$. We define a positive symmetric linear form $\langle\cdot \mid \cdot\rangle_{\Psi}$ on $\mathbb{M}(X)$ by

$$
\left\langle\sum_{i=1}^{n} r_{i} \delta_{x_{i}} \mid \sum_{j=1}^{k} s_{j} \delta_{y_{i}}\right\rangle_{\Psi}=-\frac{1}{2} \sum_{i=1}^{n} \sum_{j=1}^{k} r_{i} s_{j} \Psi\left(x_{i}, y_{j}\right) .
$$

Also, if $N_{\Psi}$ denotes the null space

$$
N_{\Psi}=\left\{\xi \in \mathbb{M}(X) \mid\langle\xi \mid \xi\rangle_{\Psi}=0\right\},
$$

then $\langle\cdot \mid \cdot\rangle_{\Psi}$ defines an inner product on the quotient $\mathbb{M}(X) / N_{\Psi}$ and we obtain a real Hilbert space $\mathcal{K}$ as the completion of $\mathbb{M}(X) / N_{\Psi}$ with respect to $\langle\cdot \mid \cdot\rangle_{\Psi}$.

We remark that, if $\Psi$ is defined by a map $\sigma: X \rightarrow \mathcal{H}$ as above and $e \in X$ is any choice of base point, the map $\phi_{e}: X \rightarrow \mathcal{K}$ defined by $\phi_{e}(x)=\delta_{x}-\delta_{e}$ satisfies $\left\|\phi_{e}(x)-\phi_{e}(y)\right\|_{\mathcal{K}}=\|\sigma(x)-\sigma(y)\|_{\mathcal{H}}$. Indeed,

$$
\begin{aligned}
\left\|\phi_{e}(x)-\phi_{e}(y)\right\|_{\mathcal{K}}^{2} & =\left\langle\phi_{e}(x)-\phi_{e}(y) \mid \phi_{e}(x)-\phi_{e}(y)\right\rangle \\
& =\left\langle\delta_{x}-\delta_{y} \mid \delta_{x}-\delta_{y}\right\rangle \\
& =-\frac{1}{2}(\Psi(x, x)+\Psi(y, y)-\Psi(x, y)-\Psi(y, x)) \\
& =\Psi(x, y) \\
& =\|\sigma(x)-\sigma(y)\|_{\mathcal{H}}^{2} .
\end{aligned}
$$

Also, if $G \curvearrowright X$ is an action of a group $G$ on $X$ and $\Psi$ is $G$-invariant, that is, $\Psi(g x, g y)=\Psi(x, y)$, this action lifts to an action $\pi: G \curvearrowright \mathbb{M}(X)$ preserving the form $\langle\cdot \mid \cdot\rangle_{\Psi}$ via $\pi(g) \xi=\xi\left(g^{-1} \cdot\right)$. It follows that $\pi$ factors through to an orthogonal (i.e., isometric linear) representation $G \curvearrowright \mathcal{K}$.

A version of Lemma 5 below is originally due to Aharoni et al. [3] for the case of abelian groups and has been extended and refined several times recently in connection with the coarse geometry of Banach spaces and locally compact groups (see, e.g., [20, 33, 51]). Since more care is needed when dealing with general amenable as opposed to locally compact amenable or abelian groups, we include a full proof.

Let us first recall that, if $\sigma: X \rightarrow Y$ is a map between metric spaces, the exact compression modulus $\tilde{\kappa}$ of $\sigma$ is given by $\tilde{\kappa}(t)=\inf _{d\left(x, x^{\prime}\right)=t} d\left(\sigma(x), \sigma\left(x^{\prime}\right)\right)$.

Lemma 5. Suppose $d$ is a compatible left-invariant metric on an amenable topological group $G$ and $\sigma:(G, d) \rightarrow \mathcal{H}$ is a uniformly continuous and 
bornologous map into a Hilbert space $\mathcal{H}$ with exact compression modulus $\tilde{\kappa}$ and expansion modulus $\theta$. Then there is a continuous $G$-invariant kernel conditionally of negative type $\Psi: G \times G \rightarrow \mathbb{R}_{+}$satisfying

$$
\tilde{\kappa}(d(g, f))^{2} \leqslant \Psi(g, f) \leqslant \theta(d(g, f))^{2} .
$$

Proof. For fixed $g, h \in G$, we define a function $\phi_{g, h}: G \rightarrow \mathbb{R}$ via

$$
\phi_{g, h}(f)=\|\sigma(f g)-\sigma(f h)\|^{2} .
$$

Since, for all $g, h, f \in G$, we have

$$
\tilde{\kappa}(d(g, h))^{2}=\tilde{\kappa}(d(f g, f h))^{2} \leqslant\|\sigma(f g)-\sigma(f h)\|^{2} \leqslant \theta(d(g, h))^{2},
$$

it follows that

$$
\tilde{\kappa}(d(g, h))^{2} \leqslant \phi_{g, h} \leqslant \theta(d(g, h))^{2}
$$

and so, in particular, $\phi_{g, h} \in \ell^{\infty}(G)$.

We claim that $\phi_{g, h}$ is left-uniformly continuous, that is, that for all $\epsilon>0$ there is $W \ni 1$ open so that $\left|\phi_{g, h}(f)-\phi_{g, h}(f w)\right|<\epsilon$, whenever $f \in G$ and $w \in W$. To see this, take some $\eta>0$ so that $4 \eta\left\|\phi_{g, h}\right\|_{\infty}+4 \eta^{2}<\epsilon$ and find, by uniform continuity of $\sigma$, some open $V \ni 1$ so that $\|\sigma(f)-\sigma(f v)\|<\eta$ for all $f \in G$ and $v \in V$. Pick also $W \ni 1$ open so that $W g \subseteq g V$ and $W h \subseteq h V$. Then, if $f \in G$ and $w \in W$, there are $v_{1}, v_{2} \in V$ so that $w g=g v_{1}$ and $w h=h v_{2}$, whence

$$
\begin{aligned}
\left|\phi_{g, h}(f)-\phi_{g, h}(f w)\right| & =\left|\|\sigma(f g)-\sigma(f h)\|^{2}-\|\sigma(f w g)-\sigma(f w h)\|^{2}\right| \\
& =\left|\|\sigma(f g)-\sigma(f h)\|^{2}-\left\|\sigma\left(f g v_{1}\right)-\sigma\left(f h v_{2}\right)\right\|^{2}\right| \\
& <4 \eta\left\|\phi_{g, h}\right\|_{\infty}+4 \eta^{2} \\
& <\epsilon .
\end{aligned}
$$

Thus, every $\phi_{g, h}$ belongs to the closed linear subspace $\operatorname{LUC}(G) \subseteq \ell^{\infty}(G)$ of left-uniformly continuous bounded real-valued functions on $G$ and a similar calculation shows that the map $(g, h) \in G \times G \mapsto \phi_{g, h} \in \ell^{\infty}(G)$ is continuous.

Now, since $G$ is amenable, there exists a mean $\mathfrak{m}$ on $\operatorname{LUC}(G)$ invariant under the right-regular representation $\rho: G \curvearrowright \operatorname{LUC}(G)$ given by $\rho(g)(\phi)=\phi(\cdot g)$. Using this, we can define a continuous kernel $\Psi: G \times G \rightarrow \mathbb{R}$ by

$$
\Psi(g, h)=\mathfrak{m}\left(\phi_{g, h}\right)
$$

and note that $\Psi(f g, f h)=\mathfrak{m}\left(\phi_{f g, f h}\right)=\mathfrak{m}\left(\rho(f)\left(\phi_{g, h}\right)\right)=\mathfrak{m}\left(\phi_{g, h}\right)=\Psi(g, h)$ for all $g, h, f \in G$. 
We claim that $\Psi$ is a kernel conditionally of negative type. To verify this, let $g_{1}, \ldots, g_{n} \in G$ and $r_{1}, \ldots, r_{n} \in \mathbb{R}$ with $\sum_{i=1}^{n} r_{i}=0$. Then, for all $f \in G$,

$$
\sum_{i=1}^{n} \sum_{j=1}^{n} r_{i} r_{j} \phi_{g_{i}, g_{j}}(f)=\sum_{i=1}^{n} \sum_{j=1}^{n} r_{i} r_{j}\left\|\sigma\left(f g_{i}\right)-\sigma\left(f g_{j}\right)\right\|^{2} \leqslant 0,
$$

since $(g, h) \mapsto\|\sigma(f g)-\sigma(f h)\|^{2}$ is a kernel conditionally of negative type. As $\mathfrak{m}$ is positive, it follows that also

$$
\sum_{i=1}^{n} \sum_{j=1}^{n} r_{i} r_{j} \Psi\left(g_{i}, g_{j}\right)=\mathfrak{m}\left(\sum_{i=1}^{n} \sum_{j=1}^{n} r_{i} r_{j} \phi_{g_{i}, g_{j}}\right) \leqslant 0 .
$$

Finally, as $\mathfrak{m}$ is a mean and

$$
\tilde{\kappa}(d(g, h))^{2} \leqslant \phi_{g, h} \leqslant \theta(d(g, h))^{2},
$$

it follows that

$$
\tilde{\kappa}(d(g, h))^{2} \leqslant \Psi(g, h) \leqslant \theta(d(g, h))^{2}
$$

as required.

THEOREM 13. Suppose $d$ is a compatible left-invariant metric on an amenable topological group $G$ and $\sigma:(G, d) \rightarrow \mathcal{H}$ is a uniformly continuous and bornologous map into a Hilbert space $\mathcal{H}$ with exact compression modulus $\tilde{\kappa}$ and expansion modulus $\theta$.

Then there is a continuous cocycle into a real Hilbert space $b: G \rightarrow \mathcal{K}$ so that

$$
\tilde{\kappa}(d(g, f)) \leqslant\|b(g)-b(f)\| \leqslant \theta(d(g, f)),
$$

for all $g, f \in G$.

Proof. Let $\Psi$ be the $G$-invariant kernel conditionally of negative type given by Lemma 5. As above, we define a positive symmetric form $\langle\cdot \mid \cdot\rangle_{\Psi}$ on $\mathbb{M}(G)$. Note that, since $\Psi$ is $G$-invariant, the form $\langle\cdot \mid \cdot\rangle_{\Psi}$ is invariant under the leftregular representation $\lambda: G \curvearrowright \mathbb{M}(G)$ given by $\lambda(g)(\xi)=\xi\left(g^{-1} \cdot\right)$ and so $\lambda$ induces a strongly continuous orthogonal representation $\pi$ of $G$ on the Hilbert space completion $\mathcal{K}$ of $\mathbb{M}(G) / N_{\Psi}$.

Moreover, as is easily checked, the map $b: G \rightarrow \mathcal{K}$ given by $b(g)=\left(\delta_{g}-\delta_{1}\right)$ $+N_{\Psi}$ is a cocycle for $\pi$. Now

$$
\|b(g)\|^{2}=\left\langle\delta_{g}-\delta_{1} \mid \delta_{g}-\delta_{1}\right\rangle_{\Psi}=\Psi(g, 1),
$$


whence

$$
\tilde{\kappa}(d(g, 1)) \leqslant\|b(g)\| \leqslant \theta(d(g, 1)) .
$$

Replacing $g$ with $f^{-1} g$, the theorem follows.

The first application concerns uniform embeddability of balls. Recall, for example, that a Banach space $X$ whose unit ball $B_{X}$ is uniformly embeddable into a Hilbert space must have finite cotype [52, Proposition 5.3].

Corollary 3. Let $\sigma: X \rightarrow \mathcal{H}$ be a uniformly continuous map from a Banach space $X$ into a Hilbert space satisfying

$$
\inf _{\|x-y\|=r}\|\sigma(x)-\sigma(y)\|>0
$$

for just some $r>0$. Then $B_{X}$ is uniformly embeddable into Hilbert space.

Proof. Observe that the exact compression modulus of $\sigma$ satisfies $\tilde{\kappa}_{\sigma}(r)>0$. Thus, applying Theorem 13, we obtain a Hilbert valued continuous cocycle $b: X \rightarrow \mathcal{K}$ with $\tilde{\kappa}_{b}(r)>0$. Finally, an application of Proposition 2, shows that $B_{X}$ uniformly embeds into $B_{\mathcal{K}}$.

The requirement that $\sigma$ be uniformly continuous in Theorem 13 above is somewhat superfluous. Indeed, Johnson and Randrianarivony [33, Step 0] have shown that any separable Banach space admitting a coarse embedding into a Hilbert space also has a uniformly continuous coarse embedding into a Hilbert space and the proof carries over directly to prove the following.

Lemma 6 [33]. Suppose $\sigma:(X, d) \rightarrow \mathcal{H}$ is a map from a metric space into a Hilbert space and assume that $\sigma$ is Lipschitz for large distances and expanding. Then $(X, d)$ admits a uniformly continuous coarse embedding into a Hilbert space.

For a topological group, the question is then when one may work with Lipschitz for large distance maps rather than bornologous maps.

Lemma 7. Let $\sigma: G \rightarrow(X, d)$ be a bornologous map from a Polish group into a metric space. Then there is a compatible left-invariant metric $\partial$ on $G$ so that

$$
\sigma:(G, \partial) \rightarrow(X, d)
$$

is Lipschitz for large distances. 
Proof. For $a \in G$, set

$$
w(a)=\sup _{x^{-1} y=a} d(\sigma(x), \sigma(y))
$$

and observe that, as $\sigma$ is bornologous, $w(a)=w\left(a^{-1}\right)<\infty$. We claim that there is some symmetric open identity neighbourhood $V$ so that

$$
C=\sup _{a \in V} w(a)<\infty
$$

If not, there are $a_{n} \rightarrow 1$ so that $w\left(a_{n}\right)>n$. But then $K=\left\{a_{n}, 1\right\}_{n}$ is a compact and thus relatively $(\mathrm{OB})$ set and

$$
E_{K}=\left\{(x, y) \in G \times G \mid x^{-1} y \in K\right\}
$$

a coarse entourage on $G$. As $\sigma$ is bornologous, we see that $(\sigma \times \sigma) E_{K}$ is a coarse entourage in $X$, that is, $\sup _{x^{-1} y \in K} d(\sigma(x), \sigma(y))<\infty$, contradicting the assumptions on $K$.

So pick $V$ as claimed and let $D \leqslant 1$ be some compatible left-invariant metric on $G$. Fix also $\epsilon>0$ so that $V$ contains the ball $B_{D}(2 \epsilon)$. Define

$$
\begin{array}{r}
\partial(x, y)=\inf \left(\sum_{i \in B} D\left(a_{i}, 1\right)+\sum_{i \notin B}\left(w\left(a_{i}\right)+1\right) \mid x\right. \\
\left.=y a_{1} \cdots a_{n} \& a_{i} \in V \quad \text { for } i \in B\right) .
\end{array}
$$

Since $D$ is a compatible metric and $V \ni 1$ is open, $\partial$ is a continuous left-invariant écart. Moreover, as $\partial \geqslant D$, we see that $\partial$ is a compatible metric on $G$.

Now, suppose $x, y \in G$ are given and write $x=y a_{1} \cdots a_{n}$ for some $a_{i} \in G$ with $a_{i} \in V$ for $i \in B$ so that $\sum_{i \in B} D\left(a_{i}, 1\right)+\sum_{i \notin B}\left(w\left(a_{i}\right)+1\right) \leqslant \partial(x, y)+\epsilon$. Observe that, if $D\left(a_{i}, 1\right)<\epsilon$ and $D\left(a_{i+1}, 1\right)<\epsilon$ for some $i<n$, then $a_{i} a_{i+1} \in V$, so, by coalescing $a_{i}$ and $a_{i+1}$ into a single term $a_{i} a_{i+1} \in V$, we only decrease the final sum. We may therefore assume that, for every $i<n$, either $D\left(a_{i}, 1\right) \geqslant \epsilon$ or $D\left(a_{i+1}, 1\right) \geqslant \epsilon$. It thus follows that

$$
\partial(x, y)+\epsilon \geqslant \sum_{i \in B} D\left(a_{i}, 1\right)+\sum_{i \notin B}\left(w\left(a_{i}\right)+1\right) \geqslant \sum_{i \leqslant n} D\left(a_{i}, 1\right) \geqslant \frac{n-1}{2} \cdot \epsilon,
$$

that is, $n \leqslant 2 \partial(x, y) / \epsilon+3$. 
Therefore,

$$
\begin{aligned}
d(\sigma(x), \sigma(y)) \leqslant & \sum_{i \in B} d\left(\sigma\left(y a_{1} \cdots a_{i}\right), \sigma\left(y a_{1} \cdots a_{i-1}\right)\right) \\
& +\sum_{i \notin B} d\left(\sigma\left(y a_{1} \cdots a_{i}\right), \sigma\left(y a_{1} \cdots a_{i-1}\right)\right) \\
\leqslant & C \cdot n+\sum_{i \notin B} w\left(a_{i}\right) \\
\leqslant & \left(\frac{2 C}{\epsilon}+1\right) \cdot \partial(x, y)+3 C+\epsilon .
\end{aligned}
$$

In other words, $\sigma:(G, \partial) \rightarrow(X, d)$ is Lipschitz for large distances.

We can now extend the definition of the Haagerup property (see, e.g., [9, Definition 2.7.5] or [18]) from locally compact groups to the category of all topological groups.

DEFINITION 5. A topological group $G$ is said to have the Haagerup property if it admits a coarsely proper continuous affine isometric action on a Hilbert space.

Thus, based on Theorem 13 and Lemmas 6 and 7, we have the following reformulation of the Haagerup property for amenable Polish groups.

THEOREM 14. The following conditions are equivalent for an amenable Polish group $G$ :

(1) G coarsely embeds into a Hilbert space;

(2) G has the Haagerup property.

Proof. (2) $\Rightarrow$ (1): If $\alpha: G \curvearrowright \mathcal{H}$ is a coarsely proper continuous affine isometric action, with corresponding cocycle $b: G \rightarrow \mathcal{H}$, then $b: G \rightarrow \mathcal{H}$ is a uniformly continuous coarse embedding.

(1) $\Rightarrow$ (2): If $\eta: G \rightarrow \mathcal{H}$ is a coarse embedding, then by Lemma 7 there is a compatible left-invariant metric $d$ on $G$ so that $\eta:(G, d) \rightarrow \mathcal{H}$ is Lipschitz for large distances. Since $\eta$ is a coarse embedding, it must be expanding with respect to the metric $d$. It follows from Lemma 6 that $\eta:(G, d) \rightarrow \mathcal{H}$ may also be assumed to be uniformly continuous. Thus, by Theorem 13 , there is a continuous affine isometric action $\alpha: G \curvearrowright \mathcal{K}$ on a Hilbert space $\mathcal{K}$ with associated cocycle $b: G \rightarrow \mathcal{K}$ so that, for all $g \in G$,

$$
\kappa_{\eta}(d(g, f)) \leqslant\|b(g)-b(f)\| \leqslant \theta_{\eta}(d(g, f)) .
$$


Since $\eta$ is a coarse embedding, the cocycle $b: G \rightarrow \mathcal{K}$ is coarsely proper and so is the action $\alpha$.

Haagerup [29] initially showed that finitely generated free groups have the Haagerup property. It is also known that amenable locally compact groups [8] (see also [18]) have the Haagerup property. However, this is not the case for amenable Polish groups. For example, a separable Banach space not coarsely embedding into Hilbert space such as $c_{0}$ of course also fails the Haagerup property.

There is also a converse to this. Namely, Guentner and Kaminker [28] showed that, if a finitely generated discrete group $G$ admits a affine isometric action on a Hilbert space whose cocycle $b$ grows faster than the square root of the word length, then $G$ is amenable (see [20] for the generalization to the locally compact case). It is not clear what, if any, generalization of this is possible to the setting of (OB) generated Polish groups. For example, [55, Theorem 7.2], the homeomorphism group of the $n$-sphere $\mathbb{S}^{n}$ has property (OB) and thus is quasi-isometric to a point. It therefore trivially fulfils the assumptions of the Guentner-Kaminker theorem, but is not amenable.

It is by now well known that there are Polish groups admitting no nontrivial continuous unitary or, equivalently, orthogonal, representations (the first example seems to be due to Christensen and Herer [32]). Also many of these examples are amenable. While the nonexistence of unitary representations may look like a local condition on the group that can be detected in neighbourhoods of the identity, the following result shows that under extra assumptions this is also reflected in the large scale behaviour of the group.

Proposition 6. Suppose $G$ is an amenable Polish group with no nontrivial unitary representations. Then $G$ is either coarsely equivalent to a point or is not coarsely embeddable into Hilbert space.

Proof. Suppose $G$ is not coarsely equivalent to a point. Then, by Theorem 14, if $G$ is coarsely embeddable into Hilbert space, there is a coarsely proper continuous affine isometric action of $G$ on Hilbert space. So either the linear part $\pi$ is a nontrivial orthogonal representation of $G$ or the corresponding cocycle $b: G \rightarrow$ $\mathcal{H}$ is a coarsely proper continuous homomorphism. Thus, as $G$ is not coarsely equivalent to a point, in the second case, $b$ is unbounded and so composing with an appropriate linear functional, we get an unbounded homomorphism into $\mathbb{R}$. In either case, $G$ will admit a nontrivial orthogonal and thus also unitary representation contrary to our assumptions.

Finally, let us sum up the equivalences for Banach spaces. 
THEOREM 15. The following conditions are equivalent for a separable Banach space $X$ :

(1) X coarsely embeds into a Hilbert space;

(2) X uniformly embeds into a Hilbert space;

(3) X admits an uncollapsed uniformly continuous map into a Hilbert space;

(4) X has the Haagerup property.

Here Condition (4) of Theorem 15 is a priori the strongest of the four since a continuous coarsely proper cocycle $b: X \rightarrow \mathcal{H}$ will be simultaneously a uniform and coarse embedding. The equivalence of (1) and (2) was proved in [33] (the implication from (3) to (1) and (2) of course also being a direct consequence of Theorem 11), while the implication from (2) to (4) following from Theorem 14. A seminal characterization of uniform embeddability into Hilbert spaces utilizing a version of Lemma 5 appears in [3].

\section{Preservation of local structure}

Weakening the geometric restrictions on the phase space from euclidean to uniformly convex, we still have a result similar to Theorem 13. However, in this case, we do not know if amenability suffices, but must rely on strengthenings of this.

Before we state the next result, we recall that a Banach space $X$ is said to be finitely representable in a Banach space $Y$ if, for every finite-dimensional subspace $F \subseteq X$ and every $\epsilon>0$, there is a linear embedding $T: F \rightarrow Y$ so that $\|T\| \cdot\left\|T^{-1}\right\|<1+\epsilon$. We also say that $X$ is crudely finitely representable in $Y$ if there is a constant $K$ so that every finite-dimensional subspace of $X$ is $K$ isomorphic to a subspace of $Y$. Finally, if $E$ is a Banach space and $1 \leqslant p<\infty$, we let $L^{p}(E)$ denote the Banach space of equivalence classes of measurable functions $f:[0,1] \rightarrow E$ so that the $p$-norm

$$
\|f\|_{L^{p}(E)}=\left(\int_{0}^{1}\|f\|_{E}^{p} d \lambda\right)^{1 / p}
$$

is finite.

The next theorem is the basic result for preservation of local structure. Earlier versions of this are due to Naor and Peres [47] and Pestov [49] respectively for finitely generated amenable and locally finite discrete groups. 
THEOREM 16. Suppose that $d$ is a continuous left-invariant écart on a Folner amenable Polish group $G$ and $\sigma:(G, d) \rightarrow E$ is a uniformly continuous and bornologous map into a Banach space $E$ with exact compression modulus $\tilde{\kappa}$ and expansion modulus $\theta$.

Then, for every $1 \leqslant p<\infty$, there is a continuous affine isometric action of $G$ on a Banach space $V$ finitely representable in $L^{p}(E)$ with corresponding cocycle $b$ satisfying

$$
\tilde{\kappa}(d(x, y)) \leqslant\|b(x)-b(y)\|_{V} \leqslant \theta(d(x, y))
$$

for all $x, y \in G$.

Proof. Let us first assume that $G$ satisfies the second assumption of Definition 3 and let $\phi: H \rightarrow G$ be the corresponding mapping. Then, replacing $H$ by the amenable group $H / \operatorname{ker} \phi$, we may suppose that $\phi$ is injective. Let also $|\cdot|$ denote a right-invariant Haar measure on $H$. Since $H$ is amenable and locally compact, there is a Følner sequence $\left\{F_{n}\right\}$, that is, a sequence of Borel subsets $F_{n} \subseteq H$ of finite positive measure so that $\lim _{n}\left(\left|F_{n} x \triangle F_{n}\right| /\left|F_{n}\right|\right)=0$ for all $x \in H$. Let also $L^{p}\left(F_{n}, E\right)$ denote the Banach space of $p$-integrable functions $f: F_{n} \rightarrow E$ with norm

$$
\|f\|_{L^{p}\left(F_{n}, E\right)}=\left(\int_{F_{n}}\|f(x)\|_{E}^{p}\right)^{1 / p} .
$$

Now fix a nonprincipal ultrafilter $\mathcal{U}$ on $\mathbb{N}$ and let $\left(\prod_{n} L^{p}\left(F_{n}, E\right)\right)_{\mathcal{U}}$ denote the corresponding ultraproduct. That is, if we equip

$$
W=\left\{\left(\xi_{n}\right) \in \prod_{n} L^{p}\left(F_{n}, E\right) \mid \sup _{n}\left\|\xi_{n}\right\|_{L^{p}\left(F_{n}, E\right)}<\infty\right\}
$$

with the seminorm

$$
\left\|\left(\xi_{n}\right)\right\|_{\mathcal{U}}=\lim _{\mathcal{U}}\left\|\xi_{n}\right\|_{L^{p}\left(F_{n}, E\right)}
$$

and let $N=\left\{\left(\xi_{n}\right) \in W \mid\left\|\left(\xi_{n}\right)\right\|_{W}=0\right\}$ denote the corresponding null space, then $\left(\prod_{n} \ell^{p}\left(F_{n}, E\right)\right)_{\mathcal{U}}$ is the quotient $W / N$. For simplicity of notation, if $\left(\xi_{n}\right) \in W$, we denote the element $\left(\xi_{n}\right)+N \in\left(\prod_{n} L^{p}\left(F_{n}, E\right)\right)_{\mathcal{U}}$ by $\left(\xi_{n}\right)_{\mathcal{U}}$.

Consider the linear operator $\Theta: L^{\infty}(H, E) \rightarrow\left(\prod_{n} L^{p}\left(F_{n}, E\right)\right)_{\mathcal{U}}$ given by

$$
\Theta(f)=\left(\left|F_{n}\right|^{-1 / p} \cdot f \uparrow_{F_{n}}\right)_{\mathcal{U}}
$$


Then $\|f\|_{\mathcal{U}, p}=\|\Theta(f)\|_{\mathcal{U}}$ defines a seminorm on $L^{\infty}(H, E)$ satisfying

$$
\begin{aligned}
\|f\|_{\mathcal{U}, p}^{p} & =\left\|\left(\left|F_{n}\right|^{-1 / p} \cdot f \uparrow_{F_{n}}\right) \mathcal{U}\right\|_{\mathcal{U}}^{p} \\
& =\left(\lim _{\mathcal{U}}\left\|\left|F_{n}\right|^{-1 / p} \cdot f \uparrow_{F_{n}}\right\|_{L^{p}\left(F_{n}, E\right)}\right)^{p} \\
& =\lim _{\mathcal{U}} \int_{F_{n}}\left\|\left|F_{n}\right|^{-1 / p} \cdot f(x)\right\|_{E}^{p} \\
& =\lim _{\mathcal{U}} \frac{1}{\left|F_{n}\right|} \int_{F_{n}}\|f(x)\|_{E}^{p} .
\end{aligned}
$$

We claim that $\|\cdot\|_{\mathcal{U}, p}$ is invariant under the right-regular representation $\rho: H \curvearrowright$ $L^{\infty}(H, E)$. Indeed, for all $f \in L^{\infty}(H, E)$ and $y \in H$,

$$
\begin{aligned}
\|\rho(y) f\|_{\mathcal{U}, p}^{p}-\|f\|_{\mathcal{U}, p}^{p} & =\lim _{\mathcal{U}} \frac{1}{\left|F_{n}\right|} \int_{x \in F_{n}}\|(\rho(y) f)(x)\|_{E}^{p}-\lim _{\mathcal{U}} \frac{1}{\left|F_{n}\right|} \int_{x \in F_{n}}\|f(x)\|_{E}^{p} \\
& =\lim _{\mathcal{U}} \frac{1}{\left|F_{n}\right|}\left(\int_{x \in F_{n}}\|f(x y)\|_{E}^{p}-\int_{x \in F_{n}}\|f(x)\|_{E}^{p}\right) \\
& =\lim _{\mathcal{U}} \frac{1}{\left|F_{n}\right|}\left(\int_{x \in F_{n} y}\|f(x)\|_{E}^{p}-\int_{x \in F_{n}}\|f(x)\|_{E}^{p}\right) \\
& \leqslant \lim _{\mathcal{U}} \frac{1}{\left|F_{n}\right|} \int_{x \in F_{n} y \triangle F_{n}}\|f(x)\|_{E}^{p} \\
& \leqslant\|f\|_{L^{\infty}(H, E)}^{p} \cdot \lim _{\mathcal{U}} \frac{\left|F_{n} y \triangle F_{n}\right|}{\left|F_{n}\right|} \\
& =0 .
\end{aligned}
$$

Since $\phi: H \rightarrow G$ is a homomorphism and $d$ is left-invariant, for $x, y, z \in H$ we have

$$
d(\phi(x), \phi(y))=d(\phi(z) \phi(x), \phi(z) \phi(y))=d(\phi(z x), \phi(z y))
$$

and hence

$$
\begin{aligned}
\tilde{\kappa}[d(\phi(x), \phi(y))] & =\tilde{\kappa}[d(\phi(z x), \phi(z y))] \\
& \leqslant\|\sigma \phi(z x)-\sigma \phi(z y)\|_{E} \\
& =\|(\rho(x) \sigma \phi)(z)-(\rho(y) \sigma \phi)(z)\|_{E} \\
& \leqslant \theta[d(\phi(x), \phi(y))] .
\end{aligned}
$$

This shows that, for $x, y \in H$, we have $(\rho(x) \sigma \phi)-(\rho(y) \sigma \phi) \in L^{\infty}(H, E)$ and $\tilde{\kappa}[d(\phi(x), \phi(y))]^{p} \leqslant \frac{1}{\left|F_{n}\right|} \int_{F_{n}}\|(\rho(x) \sigma \phi)-(\rho(y) \sigma \phi)\|_{E}^{p} \leqslant \theta[d(\phi(x), \phi(y))]^{p}$ 
for all $n$. By the expression for $\|\cdot\|_{\mathcal{U}, p}$, it follows that

$$
\tilde{\kappa}[d(\phi(x), \phi(y))] \leqslant\|(\rho(x) \sigma \phi)-(\rho(y) \sigma \phi)\|_{\mathcal{U}, p} \leqslant \theta[d(\phi(x), \phi(y))] .
$$

Therefore, by setting $y=1$, we see that the mapping $b: H \rightarrow L^{\infty}(H, E)$ given by

$$
b(x)=(\rho(x) \sigma \phi)-\sigma \phi
$$

is well defined. Also, as $b(x)-b(y)=(\rho(x) \sigma \phi)-(\rho(y) \sigma \phi)$, we see that

$$
\tilde{\kappa}[d(\phi(x), \phi(y))] \leqslant\|b(x)-b(y)\|_{\mathcal{U}, p} \leqslant \theta[d(\phi(x), \phi(y))] .
$$

Since $\sigma$ uniformly continuous and thus $\lim _{\epsilon \rightarrow 0_{+}} \theta(\epsilon)=0$, it follows that $b$ is uniformly continuous with respect to the metric $d(\phi(\cdot), \phi(\cdot))$ on $H$ and the seminorm $\|\cdot\|_{\mathcal{U}, p}$ on $L^{\infty}(H, E)$.

Let now $M \subseteq L^{\infty}(H, E)$ denote the null space of the seminorm $\|\cdot\|_{\mathcal{U}, p}$ and $X$ be the completion of $L^{\infty}(H, E) / M$ with respect to $\|\cdot\|_{\mathcal{U}, p}$. Clearly, $X$ is isometrically embeddable into $\left(\prod_{n} L^{p}\left(F_{n}, E\right)\right)_{\mathcal{U}}$ and the right-regular representation $\rho: H \curvearrowright$ $L^{\infty}(H, E)$ induces a linear isometric representation of $H$ on $X$, which we continue denoting $\rho$. Similarly, we view $b$ as a map into $X$.

As is easy to see, $b \in Z^{1}(H, \rho)$, that is, $b$ satisfies the cocycle identity $b(x y)=$ $\rho(x) b(y)+b(x)$ for $x, y \in H$. So, in particular, the linear span of $b[H]$ is $\rho[H]$ invariant. Moreover, since each $\rho(x) \in \rho[H]$ is an isometry, the same holds for the closed linear span $V \subseteq X$ of $b[H]$.

We claim that, for every $\xi \in V$, the map $x \mapsto \rho(x) \xi$ is uniformly continuous with respect to the metric $d(\phi(\cdot), \phi(\cdot))$. Since the linear span of $b[H]$ is dense in $V$, it suffices to prove this for $\xi \in b[H]$. So fix some $z \in H$ and note that, for $x, y \in H$, we have

$$
\begin{aligned}
\|\rho(x) b(z)-\rho(y) b(z)\|_{\mathcal{U}, p} & =\|(b(x z)-b(x))-(b(y z)-b(y))\|_{\mathcal{U}, p} \\
& \leqslant\|b(x z)-b(y z)\|_{\mathcal{U}, p}+\|b(y)-b(x)\|_{\mathcal{U}, p} \\
& \leqslant \theta[d(\phi(x z), \phi(y z))]+\theta[d(\phi(x), \phi(y))] .
\end{aligned}
$$

Now, suppose that $\epsilon>0$ is given. Then, by uniform continuity of $\sigma$, there is $\delta>0$ so that $\theta(\delta)<\epsilon / 2$. Also, since multiplication by $\phi(z)$ on the right is leftuniformly continuous on $G$, there is an $\eta>0$ so that $d(\phi(x), \phi(y))<\eta$ implies that $d(\phi(x z), \phi(y z))<\delta$. It follows that, provided $d(\phi(x), \phi(y))<\min \{\eta, \delta\}$, we have $\|\rho(x) b(z)-\rho(y) b(z)\|_{\mathcal{U}, p}<\epsilon$, hence verifying uniform continuity.

To sum up, we have now an isometric linear representation $\rho: H \curvearrowright V$ with associated cocycle $b: H \rightarrow V$ so that, with respect to the metric $d(\phi(\cdot), \phi(\cdot))$ on $H$, the mappings $b$ and $x \mapsto \rho(x) \xi$ are uniformly continuous for all $\xi \in V$.

Identifying $H$ with its image in $G$ via the continuous embedding $\phi$, it follows that there are unique continuous extensions of these mappings to all of $G$, 
which we continue denoting $b$ and $x \mapsto \rho(x) \xi$. Also, simple arguments using continuity and density show that, for all $x \in G$, the map $\xi \mapsto \rho(x) \xi$ defines a linear isometry $\rho(x)$ of $V$ so that $\rho(x y)=\rho(x) \rho(y)$ and $b(x y)=\rho(x) b(y)+$ $b(x)$. In other words, $\rho$ is a continuous isometric linear representation and $b \in Z^{1}(G, \rho)$. Moreover,

$$
\tilde{\kappa}[d(x, y)] \leqslant\|b(x)-b(y)\|_{\mathcal{U}, p} \leqslant \theta[d(x, y)]
$$

for all $x, y \in G$.

To finish the proof, it now suffices to verify that $V$ is finitely representable in $L^{p}(E)$. To see this, note that, since each $L^{p}\left(F_{n}, E\right)$ is isometrically a subspace of $L^{p}(E)$, it follows from the properties of the ultraproduct that $\left(\prod_{n} L^{p}\left(F_{n}, E\right)\right)_{\mathcal{U}}$ is finitely representable in $L^{p}(E)$. Also, by construction, $X$ and a fortiori its subspace $V$ are isometrically embeddable into $\left(\prod_{n} L^{p}\left(F_{n}, E\right)\right)_{\mathcal{U}}$, which proves the theorem under the second assumption.

Consider now instead the case when $G$ is approximately compact. The proof is very similar to the second case, so we shall just indicate the changes needed. Thus, let $K_{1} \leqslant K_{2} \leqslant K_{3} \leqslant \cdots \leqslant G$ be a chain of compact subgroups with dense union in $G$. Instead of considering the sets $F_{n}$ with the Haar measure $|\cdot|$ from $H$, we now use the $K_{n}$ with their respective Haar measures and similarly $L^{p}\left(K_{n}, E\right)$ in place of $L^{p}\left(F_{n}, E\right)$. As before, the ultraproduct $\left(\prod_{n} L^{p}\left(K_{n}, E\right)\right)_{\mathcal{U}}$ is finitely representable in $L^{p}(E)$.

Also, if $x \in \bigcup_{n} K_{n}$, then the right-regular representation $\rho(x)$ defines a linear isometry of all but finitely many $L^{p}\left(K_{n}, E\right)$, which means that $\rho(x)$ induces a linear isometry of the ultraproduct $\left(\prod_{n} L^{p}\left(K_{n}, E\right)\right)_{\mathcal{U}}$ simply by letting $\rho(x)\left(\xi_{n}\right)_{\mathcal{U}}=\left(\rho(x) \xi_{n}\right)_{\mathcal{U}}$.

Also, observe that, for $x, y \in G$, we have $\rho(x) \sigma, \rho(y) \sigma \in L^{p}\left(K_{n}, E\right)$ for all $n$ and by computations similar to those above, we find that

$$
\tilde{\kappa}[d(\phi(x), \phi(y))] \leqslant\left\|(\rho(x) \sigma-\rho(y) \sigma)_{\mathcal{U}}\right\|_{\mathcal{U}} \leqslant \theta[d(\phi(x), \phi(y))] .
$$

We may therefore define $b: G \rightarrow\left(\prod_{n} L^{p}\left(K_{n}, E\right)\right)_{\mathcal{U}}$ by letting

$$
b(x)=(\rho(x) \sigma-\sigma)_{\mathcal{U}}
$$

and note that

$$
\tilde{\kappa}[d(\phi(x), \phi(y))] \leqslant\|b(x)-b(y)\|_{\mathcal{U}} \leqslant \theta[d(\phi(x), \phi(y))] .
$$

One easily sees that, when restricted to $\bigcup_{n} K_{n}, b$ is a cocycle associated to the representation $\rho: \bigcup_{n} K_{n} \curvearrowright\left(\prod_{n} L^{p}\left(K_{n}, E\right)\right)_{\mathcal{U}}$. 
Moreover, as before, we verify that, for every $\xi \in V=\overline{b\left[\bigcup_{n} K_{n}\right]}$, the mapping $x \in \bigcup_{n} K_{n} \mapsto \rho(x) \xi \in V$ is left-uniformly continuous, so the representation $\rho$ extends to an isometric linear representation of $G$ on $V$ with associated cocycle $b$.

Naor and Peres [47] showed the above result for finitely generated discrete amenable groups using Følner sequences and asked in this connection whether one might bypass the Følner sequence of the proof and instead proceed directly from an invariant mean on the group. Of course, for a finitely generated group, this question is a bit vague since having an invariant mean or a Følner sequence are both equivalent to amenability. However, for general Polish groups this is not so and we may therefore provide a precise statement capturing the essence of their question.

Problem 1. Does Theorem 16 hold for a general amenable Polish group $G$ ?

REMARK 1. Subsequently to the appearance of the present paper in preprint, $F$. M. Schneider and A. Thom have developed a more general notion of Følner sets in topological groups [59] and were able to combine this with the mechanics of the above proof to solve Problem 1 in the affirmative. Moreover, they were also able to construct an example of an amenable Polish group, which is not Følner amenable, thereby showing that their result is indeed a proper strengthening of the above.

Our main concern here being the existence of coarsely proper affine isometric actions, let us first consider the application of Theorem 16 to that problem.

COROLlARY 4. Let $G$ be a Følner amenable Polish group admitting a uniformly continuous coarse embedding into a Banach space E. Then $G$ admits a coarsely proper continuous affine isometric action on a Banach space $V$ that is finitely representable in $L^{2}(E)$.

Though we do not in general have tools permitting us to circumvent the assumption of uniform continuity as in Lemma 6, for non-Archimedean groups we do. Indeed, assume $\sigma: G \rightarrow E$ is a coarse embedding of a non-Archimedean Polish group into a Banach space $E$. Then $G$ is locally $(\mathrm{OB})$ and thus has an open subgroup $V \leqslant G$ with property (OB) relative to $G$. Since $\sigma$ is bornologous, there is a constant $K>0$ so that $\|\sigma(g)-\sigma(f)\| \leqslant K$ whenever $f^{-1} g \in V$. Letting $X \subseteq G$ denote a set of left-coset representatives for $V$, we define $\eta(g)=\sigma(h)$, where $h \in X$ is the coset representative of $g V$. Then $\|\eta(g)-\sigma(g)\| \leqslant K$ for 
all $g \in G$, so $\eta: G \rightarrow \mathcal{H}$ is a coarse embedding and clearly constant on left cosets of $V$, whence also uniformly continuous on $G$.

COROllary 5. Let $G$ be a Følner amenable non-Archimedean Polish group admitting a coarse embedding into a Banach space E. Then $G$ admits a coarsely proper continuous affine isometric action on a Banach space $V$ that is finitely representable in $L^{2}(E)$.

Our second task is now to identify various properties of a Banach space $E$ that are inherited by any space $V$ finitely representable in $L^{2}(E)$ (or in other $L^{p}(E)$ ). Evidently, these must be local properties of Banach spaces, that is, only dependent on the class of finite-dimensional subspaces of the space in question.

We recall that a Banach space $V$ is super-reflexive if every other space crudely finitely representable in $V$ is reflexive. Since evidently $V$ is finitely representable in itself, super-reflexive spaces are reflexive. Also, every space that is crudely finitely representable in a super-reflexive space must not only be reflexive but even super-reflexive. Moreover, super-reflexive spaces are exactly those all of whose ultrapowers are reflexive. By a result of Enflo [22] (see also Pisier [50] for an improved result or [23] for a general exposition), the super-reflexive spaces can also be characterized as those admitting an equivalent uniformly convex renorming.

It follows essentially from the work of Clarkson [19] that, if $E$ is uniformly convex, then so is $L^{p}(E)$ for all $1<p<\infty$. In particular, if $E$ is super-reflexive, then so is every space finitely representable in $L^{2}(E)$.

For a second application, we shall note the preservation of Rademacher type and cotype in the above construction. For that we fix a Rademacher sequence, that is, a sequence $\left(\epsilon_{n}\right)_{n=1}^{\infty}$ of mutually independent random variables $\epsilon_{n}: \Omega \rightarrow\{-1$, $1\}$, where $(\Omega, \mathbb{P})$ is some probability space, so that $\mathbb{P}\left(\epsilon_{n}=-1\right)=\mathbb{P}\left(\epsilon_{n}=1\right)=\frac{1}{2}$. For example, we could take $\Omega=\{-1,1\}^{\mathbb{N}}$ with the usual coin tossing measure and let $\epsilon_{n}(\omega)=\omega(n)$.

Definition 6. A Banach space $X$ is said to have type $p$ for some $1 \leqslant p \leqslant 2$ if there is a constant $C$ so that

$$
\left(\mathbb{E}\left\|\sum_{i=1}^{n} \epsilon_{i} x_{i}\right\|^{p}\right)^{1 / p} \leqslant C \cdot\left(\sum_{i=1}^{n}\left\|x_{i}\right\|^{p}\right)^{1 / p}
$$

for every finite sequence $x_{1}, \ldots, x_{n} \in X$. 
Similarly, $X$ has cotype $q$ for some $2 \leqslant q<\infty$ if there is a constant $K$ so that

$$
\left(\sum_{i=1}^{n}\left\|x_{i}\right\|^{q}\right)^{1 / q} \leqslant K \cdot\left(\mathbb{E}\left\|\sum_{i=1}^{n} \epsilon_{i} x_{i}\right\|^{q}\right)^{1 / q}
$$

for every finite sequence $x_{1}, \ldots, x_{n} \in X$.

We note that, by the triangle inequality, every Banach space has type 1 . Similarly, by stipulation, every Banach space is said to have cotype $q=\infty$.

Whereas the $p$ in the formula $\left(\sum_{i=1}^{n}\left\|x_{i}\right\|^{p}\right)^{1 / p}$ is essential, this is not so with the $p$ in $\left(\mathbb{E}\left\|\sum_{i=1}^{n} \epsilon_{i} x_{i}\right\|^{p}\right)^{1 / p}$. Indeed, the Kahane-Khintchine inequality (see [5]) states that, for all $1<p<\infty$, there is a constant $C_{p}$ so that, for every Banach space $X$ and $x_{1}, \ldots, x_{n} \in X$, we have

$$
\mathbb{E}\left\|\sum_{i=1}^{n} \epsilon_{i} x_{i}\right\| \leqslant\left(\mathbb{E}\left\|\sum_{i=1}^{n} \epsilon_{i} x_{i}\right\|^{p}\right)^{1 / p} \leqslant C_{p} \cdot \mathbb{E}\left\|\sum_{i=1}^{n} \epsilon_{i} x_{i}\right\| .
$$

In particular, for any $p, q \in\left[1, \infty\left[\right.\right.$, the two expressions $\left(\mathbb{E}\left\|\sum_{i=1}^{n} \epsilon_{i} x_{i}\right\|^{p}\right)^{1 / p}$ and $\left(\mathbb{E}\left\|\sum_{i=1}^{n} \epsilon_{i} x_{i}\right\|^{q}\right)^{1 / q}$ differ at most by some fixed multiplicative constant independent of the space $X$ and the vectors $x_{i} \in X$.

Clearly, if $E$ has type $p$ or cotype $q$, then so does every space crudely finitely representable in $X$. Also, by results of Orlicz and Nordlander (see [5]), the space $L^{p}$ has type $p$ and cotype 2 , whenever $1 \leqslant p \leqslant 2$, and type 2 and cotype $p$, whenever $2 \leqslant p<\infty$. Moreover, assuming again that $E$ has type $p$ or cotype $q$, then $L^{2}(E)$ has type $p$, respectively, cotype $q$. We refer the reader to [5] for more information on Rademacher type and cotype.

By the above discussion, Corollary 4 gives us the following.

COROLlARY 6. Let $G$ be a Følner amenable Polish group admitting a uniformly continuous coarse embedding into a Banach space $E$ that is either (a) superreflexive, (b) has type $p$ or (c) cotype $q$. Then $G$ admits a coarsely proper continuous affine isometric action on a Banach space $V$ that is super-reflexive, has type $p$, respectively, has cotype $q$.

In particular, this applies when $G$ is a Banach space. Also, in fact, any combination of properties (a), (b) and (c) verified by $E$ can be preserved by $V$.

Corollary 6 applies, in particular, when $G$ admits a uniformly continuous coarse embedding into an $L^{p}$ space. In this connection, let us note that, by results of Bretagnolle et al. $[15,16]$ and Mendel and Naor [42], for $1 \leqslant q \leqslant p \leqslant \infty$, there is a map $\phi: L^{q} \rightarrow L^{p}$ which is simultaneously a uniform and coarse embedding. Thus, if $G$ admits a uniformly continuous coarse embedding into 
$L^{p}, p \leqslant 2$, then it also admits such an embedding into $L^{2}$. On the other hand, by results of Mendel and Naor [43], $L^{q}$ does not embed coarsely into $L^{p}$, whenever $\max \{2, p\}<q<\infty$.

Using Theorems 11 and 16, we conclude the following.

THEOREM 17. Let $\sigma: X \rightarrow E$ be an uncollapsed uniformly continuous map between separable Banach spaces. Then, for every $1 \leqslant p<\infty, X$ admits a coarsely proper continuous affine isometric action on a Banach space $V$ finitely representable in $L^{p}(E)$.

Also, similarly to the proof of Corollary 3, we may conclude the following.

COROLlARY 7. Let $\sigma: X \rightarrow E$ be a uniformly continuous map from a Banach space $X$ into a super-reflexive or superstable space satisfying

$$
\inf _{\|x-y\|=r}\|\sigma(x)-\sigma(y)\|>0
$$

for just some $r>0$. Then $B_{X}$ is uniformly embeddable into a super-reflexive, respectively, superstable space.

Again, [52, Proposition 5.3], in the superstable case, we may further conclude that $X$ has finite cotype. In the super-reflexive case, we may combine this with a result of Kalton to conclude the following.

COROLlary 8. Let $\sigma: X \rightarrow E$ be a uniformly continuous map from a Banach space $X$ with nontrivial type into a super-reflexive space satisfying

$$
\inf _{\|x-y\|=r}\|\sigma(x)-\sigma(y)\|>0
$$

for just some $r>0$. Then $X$ is super-reflexive.

Proof. Indeed, [34, Theorem 5.1], if $B_{X}$ is uniformly embeddable into a uniformly convex space or, equivalently, into a super-reflexive space, and $X$ has nontrivial type, then $X$ is super-reflexive. The result now follows by applying Corollary 7 .

\section{Stable metrics, wap functions and reflexive spaces}

While it would be desirable to have a result along the lines of Theorems 13 and 16 for reflexive spaces, things are more complicated here and requires auxiliary concepts, namely, stability and weakly almost periodic functions. 
Definition 7. A function $\Phi: X \times X \rightarrow \mathbb{R}$ on a set $X$ is stable provided that, for all sequences $\left(x_{n}\right)$ and $\left(y_{m}\right)$ in $X$, we have

$$
\lim _{n \rightarrow \infty} \lim _{m \rightarrow \infty} \Phi\left(x_{n}, y_{m}\right)=\lim _{m \rightarrow \infty} \lim _{n \rightarrow \infty} \Phi\left(x_{n}, y_{m}\right),
$$

whenever both limits exist in $\mathbb{R}$.

Observe that, if $\Phi$ is a metric or just an écart on $X$, then, if either of the two limits exists, both $\left(x_{n}\right)$ and $\left(y_{m}\right)$ are bounded. Thus, for an écart to be stable, it suffices to verify the criterion for bounded sequences $\left(x_{n}\right)$ and $\left(y_{m}\right)$. With this observation in mind, one can show that an écart (respectively, a bounded function) $\Phi$ is stable provided that, for all bounded $\left(x_{n}\right),\left(y_{m}\right)$ (respectively all $\left(x_{n}\right),\left(y_{m}\right)$ ), we have

$$
\lim _{n \rightarrow \mathcal{U}} \lim _{m \rightarrow \mathcal{V}} \Phi\left(x_{n}, y_{m}\right)=\lim _{m \rightarrow \mathcal{V}} \lim _{n \rightarrow \mathcal{U}} \Phi\left(x_{n}, y_{m}\right)
$$

whenever $\mathcal{U}, \mathcal{V}$ are nonprincipal ultrafilters on $\mathbb{N}$.

We remark that a simple, but tedious, inspection shows that, if $d$ is a stable écart on $X$, then so is the uniformly equivalent bounded écart $D(x, y)=$ $\min \{d(x, y), 1\}$.

In keeping with the terminology above, a Banach space $(X,\|\cdot\|)$ is stable if the norm metric is stable. This class of spaces was initially studied by Krivine and Maurey [38] in which they showed that every stable Banach space contains a copy of $\ell^{p}$ for some $1 \leqslant p<\infty$. As we shall see, for several purposes including the geometry of Banach spaces, the following more general class of groups plays a central role.

DEFINITION 8. A topological group $G$ is metrically stable if $G$ admits a compatible left-invariant stable metric.

Recall that a bounded function $\phi: G \rightarrow \mathbb{R}$ on a group $G$ is said to be weakly almost periodic (WAP) provided that its orbit $\lambda(G) \phi=\left\{\phi\left(g^{-1} \cdot\right) \mid g \in G\right\}$ under the left-regular representation $\lambda: G \curvearrowright \ell^{\infty}(G)$ is a relatively weakly compact subset of $\ell^{\infty}(G)$. The connection between stability and weak almost periodicity is provided by the following well-known criterion of Grothendieck [26].

THEOREM 18 (Grothendieck [26]). A bounded function $\phi: G \rightarrow \mathbb{R}$ on a group $G$ is weakly almost periodic if and only if the function $\Phi(x, y)=\phi\left(x^{-1} y\right)$ is stable.

There is a tight connection between weakly almost periodic functions and representations on reflexive Banach space borne out by several classical results. 
Central among these is the fact that every continuous bounded weakly almost periodic function $\phi$ on a topological group $G$ is a matrix coefficient of a strongly continuous isometric linear representation $\pi: G \curvearrowright E$ on a reflexive Banach space $E$, that is, for some $\xi \in E$ and $\eta^{*} \in E^{*}$,

$$
\phi(x)=\left\langle\pi(x) \xi, \eta^{*}\right\rangle .
$$

Conversely, every such matrix coefficient is weakly almost periodic.

We shall need some more recent results clarifying reflexive representability of Polish groups. Here a topological group $G$ is said to admit a topologically faithful isometric linear representation on a reflexive Banach space $E$ if $G$ is isomorphic to a subgroup of the linear isometry group $\operatorname{Isom}(E)$ equipped with the strong operator topology. The following theorem combines results due to Shtern [60], Megrelishvili [41] and Ben Yaacov et al. [10].

THEOREM $19[\mathbf{1 0}, \mathbf{4 1}, \mathbf{6 0}]$. For a Polish group $G$, the following are equivalent:

(1) $G$ is metrically stable;

(2) G has a topologically faithful isometric linear representation on a separable reflexive Banach space;

(3) for every identity neighbourhood $V \subseteq G$, there is a continuous weakly almost periodic function $\phi: G \rightarrow[0,1]$ so that $\phi\left(1_{G}\right)=1$ and $\operatorname{supp}(\phi)$ $\subseteq V$

In particular, the isometry group of a separable reflexive Banach space is metrically stable. Moreover, if $E$ is a stable Banach space, then $\operatorname{Isom}(E)$ is metrically stable. Indeed, by Lemma $3, \operatorname{Isom}(E)$ admits a cocycle $b: \operatorname{Isom}(E) \rightarrow$ $\ell^{2}(E)$ which is a uniform embedding. As also $\ell^{2}(E)$ is stable, it follows that $d(x, y)=\|b(x)-b(y)\|_{\ell^{2}(E)}$ is a compatible left-invariant stable metric on $\operatorname{Isom}(E)$.

Conversely, there are examples, such as the group Homeo H $_{+}[0,1]$ of increasing homeomorphisms of the unit interval [40], that admit no nontrivial continuous isometric linear actions on a reflexive space.

We now aim at extending earlier results of Raynaud [52] on the existence of $\ell^{p}$ subspaces of Banach spaces. Observe first that every left-invariant compatible metric on a Banach space is in fact bi-invariant and uniformly equivalent to the norm metric. Thus, a Banach space is metrically stable exactly when it admits an invariant stable metric uniformly equivalent to the norm. Formulated in our terminology, [52, Théorème 4.1] states the following.

THEOREM 20 (Raynaud). Every metrically stable Banach space contains an isomorphic copy of $\ell^{p}$ for some $1 \leqslant p<\infty$. 
Also, by Theorem 19 and Proposition 4, we see that a metrically stable separable Banach space admits a coarsely proper continuous affine isometric action on a separable reflexive Banach space.

Furthermore, [52, Théorème 0.2], Raynaud showed that any Banach space uniformly embeddable in a superstable Banach space, that is, a space all of whose ultrapowers are stable, is metrically stable (in fact, that it has a compatible invariant superstable metric).

Note finally that, since by [52] the class of superstable Banach spaces is closed under passing from $E$ to $L^{p}(E), 1 \leqslant p<\infty$, and under finite representability, Theorem 17 implies that a separable Banach space $E$ uniformly embeddable into a superstable Banach space also admits a coarsely proper continuous cocycle on a superstable space.

THEOREM 21. Let $X$ be a separable Banach space admitting an uncollapsed uniformly continuous map into the unit ball $B_{E}$ of a super-reflexive Banach space $E$. Then $X$ is metrically stable and contains an isomorphic copy of $\ell^{p}$ for some $1 \leqslant p<\infty$.

Proof. Let $\sigma: X \rightarrow B_{E}$ be the map in question. By replacing $E$ by the closed linear span of the image of $Y$, we can suppose that $E$ is separable. Let $\kappa$ and $\theta$ be the compression and expansion moduli of $\sigma$. As $\sigma$ is uncollapsed, $\kappa(t)>0$ for some $t>0$ and, as $\sigma$ maps into $B_{E}$, we have $\theta(s) \leqslant 2$ for all $s$. By Theorem 16, there is a Banach space $V$ finitely representable in $L^{2}(E)$ and a strongly continuous isometric linear representation $\pi: X \curvearrowright V$ with a continuous cocycle $b: X \rightarrow V$ satisfying

$$
\kappa(\|x-y\|) \leqslant\|b(x)-b(y)\|_{V} \leqslant \theta(\|x-y\|) .
$$

Being finitely representable in the super-reflexive space $L^{2}(E)$, it follows that $V$ is super-reflexive itself. Also, $b$ is bounded and uncollapsed, whence, by Proposition $1, b$ is a uniform embedding of $X$ into $V$. Being a bounded cocycle in a super-reflexive space, we conclude by the Ryll-Nardzewski fixed point theorem that $b$ is a coboundary, that is, that $b(x)=\xi-\pi(x) \xi$ for some $\xi \in V$ and all $x \in X$.

Now, since $x \mapsto \xi-\pi(x) \xi$ is a uniform embedding, so is $x \mapsto \pi(x) \xi$, which shows that $\pi: X \rightarrow \operatorname{Isom}(V)$ is a topologically faithful isometric linear representation on a reflexive Banach space. By Theorem 19, we conclude that $X$ is metrically stable. Finally, by Theorem 20, $X$ contains an isomorphic copy of $\ell^{p}$ for some $1 \leqslant p<\infty$. 
A question first raised by Aharoni [2] (see also the discussion [11, Ch. 8]) is to determine the class of Banach spaces $X$ uniformly embeddable into their unit ball $B_{X}$. It follows from [1] that $c_{0}$ embeds uniformly into $B_{c_{0}}$ and, in fact, every separable Banach space $X$ containing $c_{0}$ embeds uniformly into its ball $B_{X}$. Similarly, for $1 \leqslant p \leqslant 2, L^{p}([0,1])$ is uniformly embeddable into the unit ball of $L^{2}([0,1])$ [3]. Since also, for all $1 \leqslant p<\infty$, the unit balls of $L^{p}([0,1])$ and $L^{2}([0,1])$ are uniformly homeomorphic by a result of Odell and Schlumprecht [48], it follows that $L^{p}([0,1])$ in uniformly embeddable into the ball $B_{L^{p}([0,1])}$ for $1 \leqslant p \leqslant 2$. This fails for $p>2$ by results of [3].

COROLlaRY 9. Let $E$ be a separable super-reflexive Banach space not containing $\ell^{p}$ for any $1 \leqslant p<\infty$ or, more generally, which is not metrically stable. Then $E$ is not uniformly embeddable into $B_{E}$.

For example, this applies to the 2-convexification $T_{2}$ of the Tsirelson space and to V. Ferenczi's uniformly convex HI space [24].

One may wonder whether Theorem 21 has an analogue for uniform embeddings into super-reflexive spaces as opposed to their balls. That is, if $X$ is an infinitedimensional Banach space uniformly embeddable into a super-reflexive space, does it follow that $X$ contains an isomorphic copy of $\ell^{1}$ or an infinite-dimensional super-reflexive subspace? However, as shown by Braga [14, Corollary 4.15], Tsirelson's space $T$ uniformly embeds into the super-reflexive space $\left(T_{2}\right)_{T_{2}}$ without, of course, containing $\ell^{1}$ or a super-reflexive subspace. The best one might hope for is thus some asymptotic regularity property of $X$.

We shall now consider the existence of coarsely proper continuous affine isometric actions on reflexive spaces by potentially nonamenable groups. For locally compact second countable groups, such actions always exist, since Brown and Guentner [17] showed that every countable discrete group admits a proper affine isometric action on a reflexive Banach space and Haagerup and Przybyszewska [30] generalized this to locally compact second countable groups. Also, Kalton [34] showed that every stable metric space may be coarsely embedded into a reflexive Banach space, while, on the contrary, the Banach space $c_{0}$ does not admit a coarse embedding into a reflexive Banach space.

Our goal here is to provide an equivariant counterpart of Kalton's theorem.

THEOREM 22. Suppose a topological group $G$ carries a continuous left-invariant coarsely proper stable écart. Then $G$ admits a coarsely proper continuous affine isometric action on a reflexive Banach space.

Theorem 22 is a corollary of the following more detailed result. 
THEOREM 23. Suppose $d$ is a continuous left-invariant écart on a topological group $G$ and assume that, for all $\alpha>0$, there is a continuous weakly almost periodic function $\phi \in \ell^{\infty}(G)$ with $d$-bounded support so that $\phi \equiv 1$ on $D_{\alpha}=$ $\{g \in G \mid d(g, 1) \leqslant \alpha\}$.

Then $G$ admits a continuous isometric action $\pi: G \curvearrowright X$ on a reflexive Banach space and a continuous cocycle $b: G \rightarrow X$ that is coarsely proper with respect to the écart $d$.

Proof. Under the given assumptions, we claim that, for every integer $n \geqslant 1$, there is a continuous weakly almost periodic function $0 \leqslant \phi_{n} \leqslant 1$ on $G$ so that:

(1) $\left\|\phi_{n}\right\|_{\infty}=\phi\left(1_{G}\right)=1$;

(2) $\left\|\phi_{n}-\lambda(g) \phi_{n}\right\|_{\infty} \leqslant 1 / 4^{n}$ for all $g \in D_{n}$; and

(3) $\operatorname{supp}\left(\phi_{n}\right)$ is $d$-bounded.

To see this, we pick inductively sequences of continuous weakly almost periodic functions $\left(\psi_{i}\right)_{i=1}^{4^{n}}$ and radii $\left(r_{i}\right)_{i=0}^{4^{n}}$ so that:

(i) $0=r_{0}<2 n<r_{1}<r_{1}+2 n<r_{2}<r_{2}+2 n<r_{3}<\cdots<r_{4^{n}}$;

(ii) $0 \leqslant \psi_{i} \leqslant 1$;

(iii) $\psi_{i} \equiv 1$ on $D_{r_{i-1}+n}$;

(iv) $\operatorname{supp}\left(\psi_{i}\right) \subseteq D_{r_{i}}$.

Note first that, by the choice of $r_{i}$, the sequence

$$
D_{r_{0}+n} \backslash D_{r_{0}}, D_{r_{1}} \backslash D_{r_{0}+n}, D_{r_{1}+n} \backslash D_{r_{1}}, D_{r_{2}} \backslash D_{r_{1}+n}, \ldots, D_{r_{4} n} \backslash D_{r_{4^{n}-1}+n}, G \backslash D_{r_{4^{n}}}
$$

partitions $G$. Also, for all $1 \leqslant i \leqslant 4^{n}$,

$$
\psi_{1} \equiv \cdots \equiv \psi_{i} \equiv 0, \text { while } \psi_{i+1} \equiv \cdots \equiv \psi_{4^{n}} \equiv 1 \text { on } D_{r_{i}+n} \backslash D_{r_{i}}
$$

and

$$
\psi_{1} \equiv \cdots \equiv \psi_{i-1} \equiv 0, \text { while } \psi_{i+1} \equiv \cdots \equiv \psi_{4^{n}} \equiv 1 \text { on } D_{r_{i}} \backslash D_{r_{i-1}+n} .
$$

Setting $\phi_{n}=\left(1 / 4^{n}\right) \sum_{i=1}^{4^{n}} \psi_{i}$, we note that, for all $1 \leqslant i \leqslant 4^{n}$,

$$
\phi_{n} \equiv \frac{4^{n}-i}{4^{n}} \quad \text { on } D_{r_{i}+n} \backslash D_{r_{i}}
$$


and

$$
\frac{4^{n}-i}{4^{n}} \leqslant \phi_{n} \leqslant \frac{4^{n}-i+1}{4^{n}} \quad \text { on } D_{r_{i}} \backslash D_{r_{i-1}+n} .
$$

Now, if $g \in D_{n}$ and $f \in G$, then $\left|d\left(g^{-1} f, 1\right)-d(f, 1)\right|=\mid d(f, g)-d(f$, $1) \mid \leqslant d(g, 1) \leqslant n$. So, if $f$ belongs to some term in the above partition, then $g^{-1} f$ either belongs to the immediately preceding, the same or the immediately following term of the partition. By the above estimates on $\phi_{n}$, it follows that $\left|\phi_{n}(f)-\phi_{n}\left(g^{-1} f\right)\right| \leqslant 1 / 4^{n}$. In other words, for $g \in D_{n}$, we have

$$
\left\|\phi_{n}-\lambda(g) \phi_{n}\right\|_{\infty}=\sup _{f \in G}\left|\phi_{n}(f)-\phi_{n}\left(g^{-1} f\right)\right| \leqslant \frac{1}{4^{n}},
$$

which verifies condition (2). Conditions (1) and (3) easily follow from the construction.

Consider now a specific $\phi_{n}$ as above and define

$$
W_{n}=\overline{\operatorname{conv}}\left(\lambda(G) \phi_{n} \cup-\lambda(G) \phi_{n}\right) \subseteq B_{\ell^{\infty}(G)}
$$

and, for every $k \geqslant 1$,

$$
U_{n, k}=2^{k} W_{n}+2^{-k} B_{\ell^{\infty}(G)} .
$$

Let $\|\cdot\|_{n, k}$ denote the gauge on $\ell^{\infty}(G)$ defined by $U_{n, k}$, that is,

$$
\|\psi\|_{n, k}=\inf \left(\alpha>0 \mid \psi \in \alpha \cdot U_{n, k}\right) .
$$

If $g \in D_{n}$, then $\left\|\phi_{n}-\lambda(g) \phi_{n}\right\|_{\infty} \leqslant 1 / 4^{n}$ and so, for $k \leqslant n$,

$$
\phi_{n}-\lambda(g) \phi_{n} \in \frac{1}{2^{n}} \cdot 2^{-k} B_{\ell^{\infty}(G)} \subseteq \frac{1}{2^{n}} \cdot U_{n, k}
$$

In particular,

$$
\left\|\phi_{n}-\lambda(g) \phi_{n}\right\|_{n, k} \leqslant \frac{1}{2^{n}}, \quad \text { for all } k \leqslant n \text { and } g \in D_{n} .
$$

On the other hand, for all $g \in G$ and $k$, we have $\phi_{n}-\lambda(g) \phi_{n} \in 2 W_{n} \subseteq$ $\left(1 / 2^{k-1}\right) U_{n, k}$. Therefore,

$$
\left\|\phi_{n}-\lambda(g) \phi_{n}\right\|_{n, k} \leqslant \frac{1}{2^{k-1}}, \quad \text { for all } k \text { and } g .
$$

Finally, since $U_{n, 1} \subseteq 2 B_{\ell^{\infty}(G)}+\frac{1}{2} B_{\ell^{\infty}(G)}=\frac{5}{2} B_{\ell^{\infty}(G)}$, we have $\|\cdot\|_{\infty} \leqslant \frac{5}{2}\|\cdot\|_{n, 1}$. So, if $g \notin\left(\operatorname{supp} \phi_{n}\right)^{-1}$, then

$$
\left\|\phi_{n}-\lambda(g) \phi_{n}\right\|_{n, 1} \geqslant \frac{2}{5}, \quad \text { for all } g \notin\left(\operatorname{supp} \phi_{n}\right)^{-1} \text {. }
$$


It follows from (1) and (2) that, for $g \in D_{n}$, we have

$$
\begin{aligned}
\sum_{k}\left\|\phi_{n}-\lambda(g) \phi_{n}\right\|_{n, k}^{2} \leqslant & \underbrace{\left(\frac{1}{2^{n}}\right)^{2}+\cdots+\left(\frac{1}{2^{n}}\right)^{2}}_{n \text { times }}+\left(\frac{1}{2^{(n+1)-1}}\right)^{2}+\left(\frac{1}{2^{(n+2)-1}}\right)^{2} \\
& +\cdots \leqslant \frac{1}{2^{n-2}},
\end{aligned}
$$

while using (3) we have, for $g \notin\left(\operatorname{supp} \phi_{n}\right)^{-1}$,

$$
\sum_{k}\left\|\phi_{n}-\lambda(g) \phi_{n}\right\|_{n, k}^{2} \geqslant \frac{4}{25} \text {. }
$$

Define $\|\cdot\|_{n}$ on $\ell^{\infty}(G)$ by $\|\psi\|_{n}=\left(\sum_{k}\|\psi\|_{n, k}^{2}\right)^{1 / 2}$ and set

$$
X_{n}=\left\{\psi \in \overline{\operatorname{span}}\left(\lambda(G) \phi_{n}\right) \subseteq \ell^{\infty}(G) \mid\|\psi\|_{n}<\infty\right\} \subseteq \ell^{\infty}(G) .
$$

By the main result of Davis et al. [21], the interpolation space $\left(X_{n},\|\cdot\|_{n}\right)$ is a reflexive Banach space. Moreover, since $W_{n}$ and $U_{n, k}$ are $\lambda(G)$-invariant subsets of $\ell^{\infty}(G)$, one sees that $\|\cdot\|_{n, k}$ and $\|\cdot\|_{n}$ are $\lambda(G)$-invariant and hence we have an isometric linear representation $\lambda: G \curvearrowright\left(X_{n},\|\cdot\|_{n}\right)$.

Note that, since $\phi_{n} \in W_{n}$, we have $\phi_{n} \in X_{n}$ and can therefore define a cocycle $b_{n}: G \rightarrow X_{n}$ associated to $\lambda$ by $b_{n}(g)=\phi_{n}-\lambda(g) \phi_{n}$. By the estimates above, we have

$$
\left\|b_{n}(g)\right\|_{n}=\left\|\phi_{n}-\lambda(g) \phi_{n}\right\|_{n} \leqslant\left(\frac{1}{\sqrt{2}}\right)^{n-2}
$$

for $g \in D_{n}$, while

$$
\left\|b_{n}(g)\right\|_{n}=\left\|\phi_{n}-\lambda(g) \phi_{n}\right\|_{n} \geqslant \frac{2}{5}
$$

for $g \notin\left(\operatorname{supp} \phi_{n}\right)^{-1}$.

Let now $Y=\left(\bigoplus_{n}\left(X_{n},\|\cdot\|_{n}\right)\right)_{\ell^{2}}$ denote the $\ell^{2}$-sum of the spaces $\left(X_{n},\|\cdot\|_{n}\right)$. Let also $\pi: G \curvearrowright Y$ be the diagonal action and $b=\bigoplus b_{n}$ the corresponding cocycle. To see that $b$ is well defined, note that, for $g \in D_{n}$, we have

$$
\begin{aligned}
\|b(g)\|_{Y} & =\left(\sum_{m=1}^{\infty}\left\|b_{m}(g)\right\|_{m}^{2}\right)^{1 / 2} \\
& =\left(\text { finite }+\sum_{m=n}^{\infty}\left\|b_{m}(g)\right\|_{m}^{2}\right)^{1 / 2} \\
& \leqslant\left(\text { finite }+\sum_{m=n}^{\infty} \frac{1}{2^{m-2}}\right)^{1 / 2} \\
& <\infty
\end{aligned}
$$

so $b(g) \in Y$. 
Remark that, whenever $g \notin\left(\operatorname{supp} \phi_{n}\right)^{-1}$, we have

$$
\|b(g)\|_{Y} \geqslant(\underbrace{\left(\frac{2}{5}\right)^{2}+\cdots+\left(\frac{2}{5}\right)^{2}}_{n \text { times }})^{1 / 2} \geqslant \frac{\sqrt{n}}{3} .
$$

As ( $\left.\operatorname{supp} \phi_{n}\right)^{-1}$ is $d$-bounded, this shows that the cocycle $b: G \rightarrow Y$ is coarsely proper with respect to $d$. We leave the verification that the action is continuous to the reader.

Let us now see how to deduce Theorem 22 from Theorem 23. So fix a continuous left-invariant coarsely proper stable écart $d$ on $G$ with corresponding balls $D_{\alpha}$. Then, for every $\alpha>0$, we can define a continuous bounded weakly almost periodic function $\phi_{\alpha}: G \rightarrow \mathbb{R}$ by

$$
\phi_{\alpha}(g)=2-\max \left\{1, \min \left\{\frac{d(g, 1)}{\alpha}, 2\right\}\right\} .
$$

We note that $\phi_{\alpha}$ has $d$-bounded support, while $\phi_{\alpha} \equiv 1$ on $D_{\alpha}$, thus verifying the conditions of Theorem 23.

\section{A fixed point property for affine isometric actions}

9.1. Kalton's theorem and solvent maps. The result of this final section has a different flavour from the preceding ones, in that our focus will be on the interplay between coarse geometry and harmonic analytic properties of groups as related to fixed points of affine isometric actions. As a first step we must consider a weakening of the concept of coarse embeddings and show how it relates to work of Kalton.

If $M \subseteq \mathbb{N}$ is an infinite set and $r \geqslant 1$, let $P_{r}(M)$ be the set of all $r$-tuples $n_{1}<\cdots<n_{r}$ with $n_{i} \in M$. We define a graph structure on $P_{r}(M)$ by letting two $r$-tuples $n_{1}<\cdots<n_{r}$ and $m_{1}<\cdots<m_{r}$ be related by an edge if

$$
n_{1} \leqslant m_{1} \leqslant n_{2} \leqslant m_{2} \leqslant \cdots \leqslant n_{r} \leqslant m_{r}
$$

or vice versa. When equipped with the induced path metric, one sees that $P_{r}(M)$ is a finite-diameter metric space with the exact diameter depending on $r$.

THEOREM 24 (Kalton [34]). Suppose $r \in \mathbb{N}$ and let $E$ be a Banach space such that $E^{(2 r)}$ is separable. Then given any uncountable family $\left\{f_{i}\right\}_{i \in I}$ of bounded maps $f_{i}: P_{r}(\mathbb{N}) \rightarrow E$ and any $\epsilon>0$, there exist $i \neq j$ and an infinite subset $M \subseteq \mathbb{N}$ so that

$$
\left\|f_{i}(\sigma)-f_{j}(\sigma)\right\|<\theta_{f_{i}}(1)+\theta_{f_{j}}(1)+\epsilon
$$

for all $\sigma \in P_{r}(M)$. 
Kalton uses this result to show that $c_{0}$ neither embeds uniformly nor coarsely into a reflexive Banach space. However, a close inspection of the proof reveals a stronger result in the coarse setting.

Definition 9. A map $\phi:(X, d) \rightarrow(M, \mathcal{E})$ from a metric space $X$ to a coarse space $M$ is said to be solvent if, for every coarse entourage $E \in \mathcal{E}$ and $n \geqslant 1$, there is a constant $R$ so that

$$
R \leqslant d(x, y) \leqslant R+n \Rightarrow(\phi(x), \phi(y)) \notin E .
$$

In the case $(M, \partial)$ is a metric space, our definition becomes more transparent. Indeed $\phi$ is solvent if there are constants $R_{n}$ for all $n \geqslant 1$ so that

$$
R_{n} \leqslant d(x, y) \leqslant R_{n}+n \Rightarrow \partial(\phi(x), \phi(y)) \geqslant n .
$$

Also, in case $X$ is actually a geodesic metric space, we have the following easy reformulation.

LEMMA 8. Let $(X, d)$ be a geodesic metric space of infinite diameter and suppose that $\phi:(X, d) \rightarrow(M, \partial)$ is a bornologous map into a metric space $(M, \partial)$. Then $\phi$ is solvent if and only if

$$
\sup _{t} \tilde{\kappa}_{\phi}(t)=\sup _{t} \inf _{d(x, y)=t} \partial(\phi(x), \phi(y))=\infty .
$$

Proof. Suppose that the second condition holds and find constants $t_{n}$ with

$$
\inf _{d(x, y)=t_{n}} \partial(\phi(x), \phi(y)) \geqslant n .
$$

Since $\phi$ is bornologous and $X$ geodesic, $\phi$ is Lipschitz for large distances and hence

$$
\partial(\phi(x), \phi(y)) \leqslant K \cdot d(x, y)+K
$$

for some constant $K$ and all $x, y \in X$.

Now, assume $t_{n^{2}} \leqslant d(x, y) \leqslant t_{n^{2}}+n$. Then, as $X$ is geodesic, there is some $z \in X$ with $d(x, z)=t_{n^{2}}$, while $d(z, y) \leqslant n$. It follows that $\partial(\phi(x), \phi(z)) \geqslant n^{2}$, while $\partial(\phi(z), \phi(y)) \leqslant K n+K$, that is,

$$
\partial(\phi(x), \phi(y)) \geqslant n^{2}-K n-K \geqslant n
$$

provided $n$ is sufficiently large. Setting $R_{n}=t_{n^{2}}$, we see that $\phi$ is solvent.

The converse is proved by noting that every distance is attained in $X$. 
While a solvent map $\phi: X \rightarrow M$ remains solvent when postcomposed with a coarse embedding of $M$ into another coarse space, then dependence on $X$ is somewhat more delicate. For this and ulterior purposes, we need the notion of near isometries.

Definition 10. A map $\phi: X \rightarrow Y$ between metric spaces $X$ and $Y$ is said to be a near isometry if there is a constant $K$ so that, for all $x, x^{\prime} \in X$,

$$
d\left(x, x^{\prime}\right)-K \leqslant d\left(\phi(x), \phi\left(x^{\prime}\right)\right) \leqslant d\left(x, x^{\prime}\right)+K .
$$

It is fairly easy to find a near isometry $\phi: X \rightarrow X$ of a metric space $X$ that is not close to any isometry of $X$, that is, so that $\sup _{x} d(\phi(x), \psi(x))=\infty$ for any isometry $\psi$ of $X$. However, in the case of Banach spaces, positive results do exist. Indeed, by the work of several authors, in particular, Gruber [27] and Gervirtz [25], if $\phi: X \rightarrow Y$ is a surjective near isometry between Banach spaces $X$ and $Y$ with defect $K$ as above and $\phi(0)=0$, then there is surjective linear isometry $U: X \rightarrow Y$ with $\sup _{x \in X}\|\phi(x)-U(x)\| \leqslant 4 K$ (see [11, Theorem 15.2]).

Now, suppose instead that $\phi$ neither surjective nor is $\phi(0)=0$, but only that $\phi K$-cobounded, that is, $\sup _{y \in Y} \inf _{x \in X}\|y-\phi(x)\| \leqslant K$. Then $Y$ has density character at most that of $X$ and hence cardinality at most that of $X$, whence a short argument shows that there is a surjective map $\psi: X \rightarrow Y$ with $\psi(0)=0$ so that $\sup _{x \in X}\|\psi(x)+\phi(0)-\phi(x)\| \leqslant 42 K$. In particular, $\psi$ is a near isometry with defect $85 K$, whence there is a surjective linear isometry $U: X \rightarrow Y$ with $\sup _{x \in X}\|\psi(x)-U(x)\| \leqslant 340 K$. All in all, we find that

$$
\sup _{x \in X}\|A(x)-\phi(x)\| \leqslant 382 K
$$

where $A: X \rightarrow Y$ is the surjective affine isometry $A=U+\phi(0)$. In other words, every cobounded near isometry $\phi: X \rightarrow Y$ is close to a surjective affine isometry $A: X \rightarrow Y$.

Let us also note the following straightforward fact.

LEMMA 9. Let

$$
X \stackrel{\sigma}{\longrightarrow} Y \stackrel{\phi}{\longrightarrow} Z \stackrel{\psi}{\longrightarrow} W
$$

be maps between metric spaces $X, Y$ and coarse spaces $Z, W$ so that $\sigma$ is a near isometry, $\phi$ is solvent and $\psi$ is a coarse embedding. Then the composition $\psi \phi \sigma: X \rightarrow W$ is also solvent.

To gauge of how weak the existence of solvable maps is, we may reutilize the ideas of Section 3. 
Proposition 7. Suppose $X$ and $E$ are Banach spaces and that there is no uniformly continuous solvent map $\psi: X \rightarrow \ell^{2}(E)$. Then, for every uniformly continuous map $\phi: X \rightarrow E$, we have

$$
\sup _{r} \inf _{\|x-y\|=r}\|\phi(x)-\phi(y)\|=0 .
$$

Proof. Assume for a contradiction that $\phi: X \rightarrow E$ is a uniformly continuous map and that $r>0$ satisfies $\inf _{\|x-y\|=r}\|\phi(x)-\phi(y)\|=\delta>0$. Without loss of generality, we may assume that $\phi(0)=0$.

Now fix $n \geqslant 1$ and choose $\epsilon_{n}>0$ small enough that $\theta_{\phi}\left(\epsilon_{n}\right) \leqslant \delta / n 2^{n}$, where $\theta_{\phi}$ is the expansion modulus of $\phi$. Set also $\psi_{n}(x)=(n / \delta) \phi\left(\left(\epsilon_{n} / n\right) x\right)$. Then

$$
\begin{aligned}
\|x-y\| \leqslant n & \Rightarrow\left\|\phi\left(\frac{\epsilon_{n}}{n} x\right)-\phi\left(\frac{\epsilon_{n}}{n} y\right)\right\| \leqslant \frac{\delta}{n 2^{n}} \\
& \Rightarrow\left\|\psi_{n}(x)-\psi_{n}(y)\right\| \leqslant \frac{1}{2^{n}},
\end{aligned}
$$

while

$$
\|x-y\|=\frac{r n}{\epsilon_{n}} \Rightarrow\left\|\psi_{n}(x)-\psi_{n}(y)\right\| \geqslant n
$$

Finally, let $\psi: X \rightarrow \ell^{2}(E)$ be defined by $\psi(x)=\left(\psi_{1}(x), \psi_{2}(x), \ldots\right)$. Then the above inequalities show that $\psi$ is well defined and uniformly continuous and also that, for every $n \geqslant 1$, there is some $R$ with

$$
\|x-y\|=R \Rightarrow\left\|\psi_{n}(x)-\psi_{n}(y)\right\|_{E} \geqslant n \Rightarrow\|\psi(x)-\psi(y)\|_{\ell^{2}(E)} \geqslant n .
$$

Applying Lemma 8, we conclude that $\psi$ is solvent, contradicting our assumptions.

We now come to the improved statement of Kalton's theorem.

THEOREM 25. Every bornologous map $\phi: c_{0} \rightarrow$ E into a reflexive Banach space $E$ is insolvent.

Proof. Let $\left(e_{k}\right)_{k=1}^{\infty}$ denote the canonical unit vector basis for $c_{0}$. If $a \subseteq \mathbb{N}$ is a finite subset, we let $\chi_{a}=\sum_{k \in a} e_{k}$. Now, for $r \geqslant 1$ and $A \subseteq \mathbb{N}$ infinite, define

$$
f_{r, A}\left(n_{1}, \ldots, n_{r}\right)=\sum_{i=1}^{r} \chi_{A \cap\left[1, n_{i}\right]} .
$$


Note that, if $n_{1} \leqslant m_{1} \leqslant n_{2} \leqslant m_{2} \leqslant \cdots \leqslant n_{r} \leqslant m_{r}$ with $n_{1}<n_{2} \cdots<n_{r}$ and $m_{1}<m_{2}<\cdots<m_{r}$, then $\left\|f_{r, A}\left(n_{1}, \ldots, n_{r}\right)-f_{r, A}\left(m_{1}, \ldots, m_{r}\right)\right\| \leqslant 1$, that is, $\theta_{f_{r, A}}(1) \leqslant 1$ and thus also $\theta_{\phi f_{r, A}}(1) \leqslant \theta_{\phi}(1)$.

Now fix $r$. Then by Theorem 24 there are infinite subsets $A, B, M \subseteq \mathbb{N}$ so that

$$
\left\|\phi f_{r, A}(\sigma)-\phi f_{r, B}(\sigma)\right\|<\theta_{\phi f_{r, A}}(1)+\theta_{\phi f_{r, B}}(1)+1 \leqslant 2 \theta_{\phi}(1)+1 .
$$

for all $\sigma \in P_{r}(M)$. On the other hand, as $A \neq B$, there is some $\sigma \in P_{r}(M)$ so that $\left\|f_{r, A}(\sigma)-f_{r, B}(\sigma)\right\|=r$, whereby

$$
\inf _{\|x-y\|=r}\|\phi(x)-\phi(y)\| \leqslant\left\|\phi f_{r, A}(\sigma)-\phi f_{r, B}(\sigma)\right\| \leqslant 2 \theta_{\phi}(1)+1 .
$$

As any interval of the form $[R, R+n]$, for $n \geqslant 1$, contains an integral point $r$, we see that $\phi$ is insolvent.

COROLlARY 10. Every bornologous map $\phi: c_{0} \rightarrow L^{1}([0,1])$ is insolvent.

Proof. By Lemma 9, it suffices to observe that $L^{1}([0,1])$ coarsely embeds into the reflexive space $L^{2}([0,1])$, which follows from [15].

Applying Proposition 7 and the fact that $E \mapsto \ell^{2}(E)$ preserves reflexivity, we also obtain the following corollary.

COROLlary 11. Every uniformly continuous map $\phi: c_{0} \rightarrow E$ into a reflexive Banach space satisfies

$$
\sup _{r} \inf _{\|x-y\|=r}\|\phi(x)-\phi(y)\|=0 .
$$

For a thorough study of regularity properties of Banach spaces preserved under solvent maps, the reader may consult [12].

9.2. Geometric Gelfand pairs. Our next step is to identify a class of topological groups whose large scale geometry is partially preserved through images by continuous homomorphisms. This is closely related to the classical notion of Gelfand pairs.

DEFINITION 11. A geometric Gelfand pair consists of a coarsely proper continuous isometric action $G \curvearrowright X$ of a topological group $G$ on a metric space $X$ so that, for some constant $K$ and all $x, y, z, u \in X$ with $d(x, y) \leqslant d(z, u)$, there is $g \in G$ with $d(g(x), z)<K$ and $d(z, g(y))+d(g(y), u)<d(z, u)+K$. 
An immediate observation is that, if $G \curvearrowright X$ is a geometric Gelfand pair and $x, z \in X$, we may set $x=y$ and $z=u$ and thus find some $g \in G$ so that $d(g(x), z)<K$. In other words, the action is also cobounded, whereby the orbit map $g \in G \mapsto g(x) \in X$ is a coarse equivalence between $G$ and $X$ for any choice of $x \in X$.

To better understand the notion of geometric Gelfand pairs, suppose that $G \curvearrowright X$ is a coarsely proper continuous and doubly transitive isometric action on a geodesic metric space, that is, so that, for all $x, y, z, v \in X$ with $d(x, y)=$ $d(z, v)$, we have $g(x)=z$ and $g(y)=v$ for some $g \in G$. Then $G \curvearrowright X$ is a geometric Gelfand pair. Indeed, if $d(x, y) \leqslant d(z, u)$, pick by geodecity some $v \in X$ so that $d(z, v)+d(v, u)=d(z, u)$ and $d(z, v)=d(x, y)$. Then, by double transitivity, there is $g \in G$ with $g(x)=z$ and $g(y)=v$, verifying that this is a geometric Gelfand pair.

Observe here that it suffices that $X$ is geodesic with respect to its distance set $S=d(X \times X)$, that is, that, for all $x, y \in X$ and $s \in S$ with $s \leqslant d(x, y)$, there is $z \in X$ with $d(x, z)+d(z, y)=d(x, y)$ and $d(x, z)=s$.

If $K$ is a compact subgroup of a locally compact group $G$, then $(G, K)$ is said to be a Gelfand pair if the convolution algebra of compactly supported $K$-biinvariant continuous functions on $G$ is commutative (see [9, Section 3.3] or [61, Section 24.8]). A basic result due to Gelfand [61, Proposition 24.8.1] states that, if $G$ admits a involutory automorphism $\alpha$ so that $g^{-1} \in K \alpha(g) K$ for all $g \in G$, then $(G, K)$ is a Gelfand pair.

In the case above of a coarsely proper continuous and doubly transitive isometric action $G \curvearrowright X$ on a geodesic metric space, let $K=\{g \in G \mid g(x)=x\}$, which is a closed subgroup of $G$. Then, if $g, f \in G$ and $f \in K g K$, there is some $h \in K$ so that $f(x)=h g(x)$ and so

$$
d(f(x), x)=d(h g(x), x)=d\left(g(x), h^{-1}(x)\right)=d(g(x), x) .
$$

Conversely, if $g, f \in G$ and $d(f(x), x)=d(g(x), x)$, then, by double transitivity, there is some $h \in G$ with $h f(x)=g(x)$ and $h(x)=x$, that is, $g^{-1} h f \in K$ and $h \in K$ whereby $f \in K g K$. In other words,

$$
f \in K g K \Longleftrightarrow d(f(x), x)=d(g(x), x) .
$$

As $d\left(g^{-1}(x), x\right)=d(x, g(x))$, this shows that $g^{-1} \in K g K$ for all $g \in G$. So $(G$, $K$ ) fulfils the condition of Gelfand's result, except for the fact that $G$ and $K$ may not be locally compact, respectively, compact. This motivates the terminology of Definition 11.

EXAMPLE 1. Probably the simplest nontrivial example of a geometric Gelfand pair is that of the canonical action $D_{\infty} \curvearrowright \mathbb{Z}$ of the infinite dihedral group $D_{\infty}$ 
on $\mathbb{Z}$ with the euclidean metric. This action is doubly transitive and proper. As moreover $\mathbb{Z}$ is geodesic with respect to its distance set $\mathbb{N}$, we see that $D_{\infty} \curvearrowright \mathbb{Z}$ is a geometric Gelfand pair.

EXAMPLE 2. Similarly, if $\operatorname{Aff}\left(\mathbb{R}^{n}\right)=O(n) \ltimes \mathbb{R}^{n}$ denotes the group of (necessarily affine) isometries of the $n$-dimensional euclidean space, then $\operatorname{Aff}\left(\mathbb{R}^{n}\right) \curvearrowright \mathbb{R}^{n}$ is a geometric Gelfand pair.

Another class of examples are constructed as above from Banach spaces. For this, let $X$ be a separable Banach space and $\operatorname{Isom}(X)$ the group of linear isometries of $X$ equipped with the strong operator topology. As every isometry of $X$ is affine, the group of all isometries of $X$ decomposes as the semidirect product $\operatorname{Aff}(X)=$ $\operatorname{Isom}(X) \ltimes(X,+)$. Also, $(X,\|\cdot\|)$ is said to be almost transitive if the action Isom $(X) \curvearrowright X$ induces a dense orbit on the unit sphere $S_{X}$ of $X$.

Proposition 8. Let $X$ be a separable Banach space. Then $\operatorname{Aff}(X) \curvearrowright X$ is a geometric Gelfand pair if and only if $\operatorname{Isom}(X)$ has property $(O B)$ and $(X,\|\cdot\|)$ is almost transitive.

Proof. First, as shown in [57], the tautological action $\operatorname{Aff}(X) \curvearrowright X$ is coarsely proper if and only if $\operatorname{Isom}(X)$ has property $(\mathrm{OB})$. So assume that $\operatorname{Isom}(X)$ has property $(\mathrm{OB})$ and consider the transitivity condition.

We observe that, if $(X,\|\cdot\|)$ is almost transitive, then $\operatorname{Aff}(X) \curvearrowright X$ is almost doubly transitive in the sense that, for all $x, y, z, u \in X$ with $\|x-y\|=\|z-u\|$ and $\epsilon>0$ there is $g \in \operatorname{Aff}(X)$ so that $g(x)=z$ and $\|g(y)-u\|<\epsilon$. In this case, $\operatorname{Aff}(X) \curvearrowright X$ is a geometric Gelfand pair. Conversely, suppose $\operatorname{Aff}(X) \curvearrowright X$ is a geometric Gelfand pair with a defect $K$. Then, for $y, z \in S_{X}$ and $\epsilon>0$, there is some $g \in \operatorname{Aff}(X)$ so that $\|g(0)-0\|<K$ and $\|g((2 K / \epsilon) y)-(2 K / \epsilon) z\|<K$. Letting $f \in \operatorname{Isom}(X)$ be the linear isometry $f(x)=g(x)-g(0)$, we find that $\|f((2 K / \epsilon) y)-(2 K / \epsilon) z\|<2 K$, that is, $\|f(y)-z\|<\epsilon$, showing that $(X,\|\cdot\|)$ is almost transitive.

Examples of almost transitive Banach spaces include $L^{p}([0,1])$ for all $1 \leqslant$ $p<\infty$ [54] and the Gurarii space $\mathbb{G}$ [39]. Moreover, as all of these spaces are separably categorical in the sense of continuous model theory, it follows from [55, Theorem 5.2] that their linear isometry group has property $(\mathrm{OB})$. We therefore conclude that $\operatorname{Aff}\left(L^{p}([0,1])\right) \curvearrowright L^{p}([0,1])$ with $1 \leqslant p<\infty$ and $\operatorname{Aff}(\mathbb{G}) \curvearrowright \mathbb{G}$ are geometric Gelfand pairs.

EXAMPLE 3. Suppose $\Gamma$ is a countable metrically homogeneous connected graph, that is, so that any isometry $f: A \rightarrow B$ with respect to the path metric on $\Gamma$ between two finite subsets extends to a full automorphism of $\Gamma$. Then the action 
$\operatorname{Aut}(\Gamma) \curvearrowright \Gamma$ is coarsely proper [57] and metrically doubly transitive. Since also the path metric is $\mathbb{Z}$-geodesic, it follows that $\operatorname{Aut}(\Gamma) \curvearrowright \Gamma$ is a geometric Gelfand pair.

Examples of metrically homogeneous countable graphs include the $n$-regular trees $T_{n}$ for all $1 \leqslant n \leqslant \aleph_{0}$ and the integral Urysohn metric space $\mathbb{Z} \mathbb{U}$.

This latter may be described as the Fraïssé limit of all finite $\mathbb{Z}$-metric spaces (i.e., with integral distances) and plays the role of a universal object in the category of $\mathbb{Z}$-metric spaces. Alternatively, $\mathbb{Z} \mathbb{U}$ is the unique universal countable $\mathbb{Z}$-metric space so that any isometry between two finite subspaces extends to a full isometry of $\mathbb{Z} \mathbb{U}$. The rational Urysohn metric space $\mathbb{Q U}$ is described similarly with $\mathbb{Q}$ in place of $\mathbb{Z}$.

Let us also recall that, since $\mathbb{Q U}$ is countable, $\operatorname{Isom}(\mathbb{Q} \mathbb{U})$ is a Polish group when equipped with the permutation group topology, which is that obtained by declaring pointwise stabilizers to be open.

EXAMPLE 4. As shown in [57], the tautological action $\operatorname{Isom}(\mathbb{Q U}) \curvearrowright \mathbb{Q U}$ is coarsely proper. Since it is also doubly transitive and $\mathbb{Q} \mathbb{U}$ is $\mathbb{Q}$-geodesic, it follows that $\operatorname{Isom}(\mathbb{Q U}) \curvearrowright \mathbb{Q U}$ is a geometric Gelfand pair.

9.3. Nearly isometric actions and quasi-cocycles. Having identified the class of geometric Gelfand pairs, we will now show a strong geometric rigidity property for their actions.

DEFINITION 12. Let $G$ be a group and $M$ a metric space. A nearly isometric action of $G$ on $M$ is a map $\alpha: G \times M \rightarrow M$ so that, for some constant $C$ and all $g, f \in G$ and $x, y \in M$, we have

$$
d(\alpha(g, x), \alpha(g, y)) \leqslant d(x, y)+C
$$

and

$$
d(\alpha(f, \alpha(g, x)), \alpha(f g, x)) \leqslant C .
$$

Thus, if for $g \in G$ we let $\alpha(g)$ denote the map $\alpha(g, \cdot): M \rightarrow M$, we see firstly that each $\alpha(g)$ is a contraction $M \rightarrow M$ up to an additive defect $C$ and secondly that $\alpha(f) \alpha(g)$ and $\alpha(f g)$ agree as maps on $M$ up to the same additive defect $C$. Thus, a nearly isometric action needs neither be a true action nor do the maps need to be exact isometries.

Nearly isometric actions can be constructed from Banach space valued quasicocycles. 
Definition 13. A map $b: G \rightarrow E$ from a group $G$ to a Banach space $E$ is said to be a quasi-cocycle provided that there is an isometric linear representation $\pi: G \curvearrowright E$ so that

$$
\sup _{g, f \in G}\|\pi(g) b(f)+b(g)-b(g f)\|<\infty .
$$

Observe that if $b: G \rightarrow E$ is a quasi-cocycle associated to an isometric linear representation $\pi: G \curvearrowright E$, then the formula

$$
\alpha(g) x=\pi(g) x+b(g)
$$

defines a nearly isometric action of $G$ on $E$. In fact, in this case, each map $\alpha(g)$ is an actual isometry. We also have the following converse to this.

LEMmA 10. Suppose that $\alpha: G \times E \rightarrow E$ is a nearly isometric action of a group $G$ on a Banach space $E$ so that the $\alpha(g): E \rightarrow E$ are uniformly cobounded. Then $b: G \rightarrow E$ defined by $b(g)=\alpha(g) 0$ is a quasi-cocycle associated to an isometric linear representation $\pi: G \curvearrowright E$ so that

$$
\sup _{g \in G} \sup _{x \in E}\|\pi(g) x+b(g)-\alpha(g) x\|<\infty .
$$

Proof. Since the maps $\alpha(g)$ are uniformly cobounded, there is a constant $C$ larger than the defect of $\alpha$ so that $\inf _{y \in E}\|x-\alpha(g) y\|<C$ for all $g \in G$ and $x \in E$.

Given any $x \in E$, find $y \in E$ with $\|x-\alpha(1) y\| \leqslant C$ and observe that

$$
\begin{aligned}
\|\alpha(1) x-x\| & \leqslant\|\alpha(1) x-\alpha(1) y\|+C \\
& \leqslant\|\alpha(1) x-\alpha(1) \alpha(1) y\|+2 C \\
& \leqslant\|x-\alpha(1) y\|+3 C \\
& \leqslant 4 C .
\end{aligned}
$$

Thus, for all $x, y \in E$ and $g \in G$,

$$
\begin{aligned}
\|x-y\| & \leqslant\|\alpha(1) x-\alpha(1) y\|+8 C \\
& \leqslant\left\|\alpha\left(g^{-1}\right) \alpha(g) x-\alpha\left(g^{-1}\right) \alpha(g) y\right\|+10 C \\
& \leqslant\|\alpha(g) x-\alpha(g) y\|+11 C \\
& \leqslant\|x-y\|+12 C,
\end{aligned}
$$

showing that $\alpha(g)$ is a near isometry of $E$ with defect $11 C$. Hence, as observed in Section 9.1, there is a linear isometry $\pi(g)$ of $E$ so that, for $b(g)=\alpha(g) 0$ and $K=5000 C$, we have

$$
\sup _{x \in E}\|\pi(g) x+b(g)-\alpha(g) x\| \leqslant K .
$$


It follows that the map $A: G \rightarrow \operatorname{Aff}(E)$ given by $A(g) x=\pi(g) x+b(g)$ defines a rough action of $G$ on $E$ in the sense [44, Section 13.1], that is, each $A(g)$ is an affine isometry of $E$ and $\sup _{x \in E}\|A(g) A(f) x-A(g f) x\| \leqslant 4 K<$ $\infty$. In particular, $\pi: G \rightarrow \operatorname{Isom}(E)$ is an isometric linear representation of $G$ and $b: G \rightarrow E$ an associated quasi-cocycle [44, Lemma 13.1.2]. Indeed $\|\pi(g) b(f)+b(g)-b(g f)\|=\|A(g) A(f) 0-A(g f) 0\| \leqslant 4 K$, whereby

$$
\begin{aligned}
\| \pi(g) \pi(f) x- & \pi(g f) x \| \\
& \leqslant\|\pi(g)(\pi(f) x+b(f))+b(g)-(\pi(g f) x+b(g f))\|+4 K \\
& \leqslant\|A(g) A(f) x-A(g f) x\|+4 K \\
& \leqslant 8 K
\end{aligned}
$$

for all $x \in E$. As $\|\pi(g) \pi(f) x-\pi(g f) x\|$ is positive homogeneous in $x$, we see that $\pi(g) \pi(f)=\pi(g f)$ for all $g, f \in G$.

As our applications deal with topological groups, it is natural to demand that a nearly isometric action $\alpha$ respects some of the topological group structure. As continuity may be too restrictive, a weaker assumption is that some orbit map is bornologous.

LEMMA 11. Let $\alpha: G \times M \rightarrow M$ be a nearly isometric action of a Polish group. Then, if some orbit map $g \in G \mapsto \alpha(g) x \in M$ is Baire measurable, it is also bornologous.

Proof. For every $n$, let $B_{n}=\left\{g \in G \mid d(\alpha(g) x, x)<n \& d\left(\alpha\left(g^{-1}\right) x\right.\right.$, $x)<n\}$. Then $G=\bigcup_{n} B_{n}$ is a covering of $G$ by countable many Baire measurable symmetric subsets, whence by the Baire category theorem some $B_{n}$ must be nonmeagre. Applying Pettis' theorem, it follows that $B_{n} B_{n}$ is an identity neighbourhood in $G$.

Now suppose that $A \subseteq G$ is relatively (OB) and find a finite set $F \subseteq G$ and $m \geqslant 1$ so that $A \subseteq\left(F B_{n} B_{n}\right)^{m}$. Let also $K$ be a number larger than all of $n$, $\max _{f \in F} d(\alpha(f) x, x)$ and the defect of $\alpha$. Then, if $h, g \in G$ and $h^{-1} g \in A$, there are $k_{1}, \ldots, k_{2 m} \in B_{n}$ and $f_{1}, \ldots, f_{m} \in F$ with $g=h f_{1} k_{1} k_{2} h_{2} k_{3} k_{4} \cdots f_{m} k_{2 m-1} k_{2 m}$. Thus,

$$
\begin{aligned}
d(\alpha(g) x, \alpha(h) x)= & d\left(\alpha\left(h f_{1} k_{1} k_{2} \cdots f_{m} k_{2 m-1} k_{2 m}\right) x, \alpha(h) x\right) \\
\leqslant & d\left(\alpha\left(h f_{1} k_{1} k_{2} \cdots f_{m} k_{2 m-1} k_{2 m}\right) x,\right. \\
& \left.\times \alpha(h) \alpha\left(f_{1} k_{1} k_{2} \cdots f_{m} k_{2 m-1} k_{2 m}\right) x\right) \\
& +d\left(\alpha(h) \alpha\left(f_{1} k_{1} k_{2} \cdots f_{m} k_{2 m-1} k_{2 m}\right) x, \alpha(h) x\right) \\
\leqslant & d\left(\alpha\left(f_{1} k_{1} k_{2} \cdots f_{m} k_{2 m-1} k_{2 m}\right) x, x\right)+2 K
\end{aligned}
$$




$$
\begin{aligned}
\leqslant & d\left(\alpha\left(f_{1} k_{1} k_{2} \cdots f_{m} k_{2 m-1} k_{2 m}\right) x, \alpha\left(f_{1}\right) \alpha\left(k_{1} k_{2} \cdots f_{m} k_{2 m-1} k_{2 m}\right) x\right) \\
& +d\left(\alpha\left(f_{1}\right) \alpha\left(k_{1} k_{2} \cdots f_{m} k_{2 m-1} k_{2 m}\right) x, \alpha(f) x\right) \\
& +d(\alpha(f) x, x)+2 K \\
\leqslant & d\left(\alpha\left(k_{1} k_{2} \cdots f_{m} k_{2 m-1} k_{2 m}\right) x, x\right)+5 K \\
\leqslant & \cdots \\
\leqslant & (2+3 m) K
\end{aligned}
$$

That is,

$$
h^{-1} g \in A \Rightarrow d(\alpha(g) x, \alpha(h) x) \leqslant(2+3 m) K,
$$

showing that the orbit map $g \in G \mapsto \alpha(g) x \in M$ is bornologous.

In particular, since a quasi-cocycle $b: G \rightarrow E$ associated to an isometric linear representation $\pi: G \curvearrowright E$ is simply the orbit map $g \mapsto \alpha(g) 0$ of the associated rough action $\alpha(g) x=\pi(g) x+b(g)$, we have the following corollary.

Lemma 12. Let $b: G \rightarrow E$ be a Baire measurable quasi-cocycle on a Polish group $G$. Then $b$ is bornologous.

EXAMPLE 5. In a great number of mainly topological transformation groups $G$, we can remove the condition of Baire measurability from Lemmas 11 and 12 . Indeed, in the above proof, we need only that, whenever $G=\bigcup_{n} B_{n}$ is a countable increasing covering by symmetric subsets, then there are $n$ and $m$ so that $\operatorname{int}\left(B_{n}^{m}\right) \neq \emptyset$. One general family of groups satisfying this criterion are Polish groups with ample generics, that is, so that the diagonal conjugacy action $G \curvearrowright G^{n}$ has a comeagre orbit for every $n \geqslant 1$ [37]. For example, both $\operatorname{Isom}(\mathbb{Q U})$ and, by the same proof, Isom $(\mathbb{Z} \mathbb{Q})$ have ample generics, while $\operatorname{Aut}\left(T_{\aleph_{0}}\right)$ has an open subgroup with ample generics [37]. Thus, in all three cases, every quasi-cocycle defined on the group is bornologous.

Proposition 9. Suppose $G \curvearrowright X$ is a geometric Gelfand pair and that $\alpha$ is a nearly isometric action of $G$ on a metric space $M$ so that every bornologous map $X \rightarrow M$ is insolvent. If some orbit map $g \in G \mapsto \alpha(g) \xi \in M$ is bornologous, then every orbit $\alpha[G] \zeta$ is bounded.

Proof. Fix $\xi \in M$ so that the orbit map $g \in G \mapsto \alpha(g) \xi$ is bornologous and let $C$ be the defect of $\alpha$, that is, for all $g, f \in G$ and $\zeta, \eta \in M$,

$$
d_{X}(\alpha(g) \zeta, \alpha(g) \eta) \leqslant d_{X}(\zeta, \eta)+C \quad \text { and } \quad d_{X}(\alpha(f) \alpha(g) \zeta, \alpha(f g) \zeta) \leqslant C .
$$

Let also $K$ be a constant witnessing that $G \curvearrowright X$ is a geometric Gelfand pair. Fix $x \in X$ and choose for each $y \in X$ some $\gamma_{y} \in G$ such that $d_{X}\left(\gamma_{y}(x), y\right)<K$. 
Define then $\phi: X \rightarrow M$ by $\phi(y)=\alpha\left(\gamma_{y}\right) \xi$. We set $V=\left\{g \in G \mid d_{X}(g x, x)<\right.$ $3 K$ \}, which is a relatively (OB) symmetric identity neighbourhood in $G$ and let

$$
L=\sup _{f^{-1} g \in V} d_{M}(\alpha(g) \xi, \alpha(f) \xi) .
$$

We claim that $\phi$ is bornologous. Indeed, observe that the composition $y \mapsto$ $\gamma_{y}(x)$ of $\gamma: X \rightarrow G$ with the orbit map $g \in G \mapsto g(x) \in X$ will be close to the identity map on $X$. So, as the orbit map $g \mapsto g(x)$ is coarsely proper and hence expanding, this implies that $\gamma$ and thus also $\phi$ are bornologous

Now, suppose that $m \geqslant 1$ and that

$$
d_{M}(\phi(x), \phi(y)) \geqslant m+3 C+2 L+\theta_{\phi}(m+2 K)
$$

for some $y \in X$. We claim that, for all $z, u \in X$,

$$
d_{X}(x, y) \leqslant d_{X}(z, u) \leqslant d_{X}(x, y)+m \Rightarrow d_{M}(\phi(z), \phi(u)) \geqslant m .
$$

To see this, suppose that $d_{X}(x, y) \leqslant d_{X}(z, u) \leqslant d_{X}(x, y)+m$ and find some $g \in G$ so that $d_{X}(g(x), z)<K$ and $d_{X}(z, g(y))+d_{X}(g(y), u)<d_{X}(z, u)+K$, that is,

$$
\begin{aligned}
d_{X}(g(y), u) & \leqslant d_{X}(z, u)+K-d_{X}(z, g(y)) \\
& \leqslant d_{X}(x, y)+m+K-d_{X}(g(x), g(y))+K \\
& =m+2 K .
\end{aligned}
$$

In particular, $d_{M}(\phi(g y), \phi(u)) \leqslant \theta_{\phi}(m+2 K)$.

Now, $d_{X}\left(g \gamma_{x}(x), \gamma_{z}(x)\right) \leqslant d_{X}(g(x), z)+2 K<3 K$, so $\gamma_{z}^{-1} g \gamma_{x} \in V$, whence

$$
d_{M}\left(\alpha\left(g \gamma_{x}\right) \xi, \phi(z)\right)=d_{M}\left(\alpha\left(g \gamma_{x}\right) \xi, \alpha\left(\gamma_{z}\right) \xi\right) \leqslant L .
$$

Similarly, $d_{X}\left(g \gamma_{y}(x), \gamma_{g y}(x)\right) \leqslant d_{X}\left(g \gamma_{y}(x), g(y)\right)+d_{X}\left(g(y), \gamma_{g y}(x)\right)<2 K$, so $\gamma_{g y}^{-1} g \gamma_{y} \in V$, whence

$$
d_{M}\left(\alpha\left(g \gamma_{y}\right) \xi, \phi(g y)\right)=d_{M}\left(\alpha\left(g \gamma_{x}\right) \xi, \alpha\left(\gamma_{g y}\right) \xi\right) \leqslant L .
$$

Thus

$$
\begin{aligned}
d_{M}(\phi(x), \phi(y)) & =d_{M}\left(\alpha\left(\gamma_{x}\right) \xi, \alpha\left(\gamma_{y}\right) \xi\right) \\
& \leqslant d_{M}\left(\alpha\left(g^{-1}\right) \alpha\left(g \gamma_{x}\right) \xi, \alpha\left(g^{-1}\right) \alpha\left(g \gamma_{y}\right) \xi\right)+2 C \\
& \leqslant d_{M}\left(\alpha\left(g \gamma_{x}\right) \xi, \alpha\left(g \gamma_{y}\right) \xi\right)+3 C \\
& \leqslant d_{M}(\phi(z), \phi(g y))+3 C+2 L \\
& \leqslant d_{M}(\phi(z), \phi(u))+3 C+2 L+\theta_{\phi}(m+2 K),
\end{aligned}
$$

whence $d_{M}(\phi(z), \phi(u)) \geqslant m$, proving the claim. 
It follows from our claim that, if $\phi$ is unbounded, then, for every $m$, there is some $R_{m}$ so that

$$
R_{m} \leqslant d_{X}(z, u) \leqslant R_{m}+m \Rightarrow d_{M}(\phi(z), \phi(u)) \geqslant m
$$

that is, $\phi$ is solvent, which is absurd. So $\phi$ is a bounded map, which easily implies that every orbit $\alpha[G] \zeta$ is bounded in $M$.

Proposition 10. Suppose $G \curvearrowright X$ is a geometric Gelfand pair and $H a$ topological group so that every bornologous map $X \rightarrow H$ is insolvent. Then, if $\pi: G \rightarrow H$ is a continuous homomorphism, $\pi[G]$ is relatively $(O B)$ in $H$.

Proof. Pick a constant $K$ witnessing that $G \curvearrowright X$ is a geometric Gelfand pair, fix $x \in X$ and choose for every $y \in X$ some $\gamma_{y} \in G$ so that $d\left(\gamma_{y}(x), y\right)<$ $K$. As in the proof of Proposition 9, $\gamma: X \rightarrow G$ is bornologous. Finally, let $V=\left\{g \in G \mid d_{X}(g x, x)<3 K\right\}$, which is a symmetric relatively (OB) identity neighbourhood in $G$.

Now assume that $A \subseteq H$ is relatively (OB), $m \geqslant 1$ and fix a symmetric relatively (OB) set $D \subseteq G$ so that $\gamma_{v} \in \gamma_{w} D$ whenever $d(w, v) \leqslant m+2 K$. Suppose that $\gamma_{y} \notin \gamma_{x} V \pi^{-1}(A) D V$ for some $y \in X$.

Suppose that $d_{X}(x, y) \leqslant d_{X}(z, u) \leqslant d_{X}(x, y)+m$ for some $z, u \in Z$ and find some $g \in G$ so that $d_{X}(g(x), z)<K$ and $d_{X}(z, g(y))+d_{X}(g(y), u)<d_{X}(z, u)$ $+K$, that is, $d_{X}(g(y), u) \leqslant m+2 K$. In particular, $\gamma_{g y} \in \gamma_{u} D$. Also $d_{X}\left(g \gamma_{x}(x)\right.$, $\left.\gamma_{z}(x)\right) \leqslant d_{X}(g(x), z)+2 K<3 K$ and

$$
d_{X}\left(g \gamma_{y}(x), \gamma_{g y}(x)\right) \leqslant d_{X}\left(g \gamma_{y}(x), g(y)\right)+d_{X}\left(g(y), \gamma_{g y}(x)\right)<2 K,
$$

so $g \gamma_{x} \in \gamma_{z} V$ and $g \gamma_{y} \in \gamma_{g y} V$. It follows that

$$
\gamma_{x}^{-1} \gamma_{y}=\left(g \gamma_{x}\right)^{-1} g \gamma_{y} \in V \gamma_{z}^{-1} \gamma_{g y} V \subseteq V \gamma_{z}^{-1} \gamma_{u} D V
$$

and so $\pi\left(\gamma_{z}\right)^{-1} \pi\left(\gamma_{u}\right) \notin A$. In other words, for all $z, u \in X$,

$$
d_{X}(x, y) \leqslant d_{X}(z, u) \leqslant d_{X}(x, y)+m \Rightarrow \pi\left(\gamma_{z}\right)^{-1} \pi\left(\gamma_{u}\right) \notin A .
$$

This shows that, if, for all relatively (OB) sets $A \subseteq H$ and $D \subseteq G$, there is some $\gamma_{y} \notin \gamma_{x} V \pi^{-1}(A) D V$, then the map $z \in X \mapsto \pi\left(\gamma_{z}\right) \in H$ is both bornologous and solvent, which is impossible. So choose some $A$ and $D$ for which it fails. As $\operatorname{im}(\gamma)$ is cobounded in $G$, it follows that $G=V \pi^{-1}(A) U$ for some relatively (OB) set $U \subseteq G$ and hence $\pi[G]$ is included in the relatively (OB) set $\pi[V] A \pi[U]$. In particular, $\pi[G]$ is relatively $(\mathrm{OB})$ in $H$. 
We are now in a position of deducing the main application of this section. Note first that Isom( $\mathbb{Q U})$ is isomorphic to a closed subgroup of the group $S_{\infty}$ of all permutations of a countable set and so, in particular, Isom( $\mathbb{Q} \mathbb{U})$ admits continuous unitary representations given by permutations of an orthogonal basis $\left(e_{x}\right)_{x \in \mathbb{Q U}}$. Moreover, by results of [62] and [37], $\operatorname{Isom}(\mathbb{Q U})$ is approximately compact and thus amenable. As shown in [57], $\operatorname{Isom}(\mathbb{Q} \mathbb{U})$ is coarsely equivalent to $\mathbb{Q U}$ itself. So, as $c_{0}$ nearly isometrically embeds into $\mathbb{Q U}$, this shows that $\operatorname{Isom}(\mathbb{Q U})$ cannot have a coarsely proper affine isometric action on a reflexive space. This is in opposition to the result of Brown-Guentner [17] and HaagerupPrzybyszewska [30] that every locally compact second countable group admits such an action. However, our result here indicates a much higher degree of geometric incompatibility.

THEOREM 26. Let $E$ be either a reflexive Banach space or $E=L^{1}([0,1])$ and let $G$ be $\operatorname{Isom}(\mathbb{Z U})$ or $\operatorname{Isom}(\mathbb{Q U})$. Then every quasi-cocycle $b: G \rightarrow E$ is bounded. In particular, every affine isometric action $G \curvearrowright E$ has a fixed point.

Proof. Observe that there are near isometries from $c_{0}$ into both $\mathbb{Z U}$ and $\mathbb{Q U}$. It thus follows from Theorem 25, respectively Corollary 10, that every bornologous map from $\mathbb{Z} U$ or $\mathbb{Q} U$ into a reflexive space or into $L^{1}([0,1])$ is insolvent. Now, by Example 5, every quasi-cocycle on $G$ is bornologous, so it follows from Proposition 9 that every quasi-cocycle $b: G \rightarrow E$ is bounded.

Now, if $\alpha: G \curvearrowright E$ is an affine isometric action, then the associated cocycle in bounded and thus the affine action has a bounded orbit. Thus, by the RyllNardzewski fixed point theorem [58] for the reflexive case or the fixed point theorem of Bader et al. [6] for $E=L^{1}([0,1])$, there is a fixed point in $E$.

Theorem 26 is motivated by questions pertaining to the characterization of property $(\mathrm{OB})$ among non-Archimedean Polish groups. Every continuous affine isometric action of a Polish group with property $(\mathrm{OB})$ on a reflexive space has a fixed point and one may ask if this characterizes property $(\mathrm{OB})$ for nonArchimedean Polish groups, that is, closed subgroups of $S_{\infty}$. As $\operatorname{Isom}(\mathbb{Q U})$ acts transitively on the infinite-diameter metric space $\mathbb{Q} U$, it fails property $(\mathrm{OB})$ and so the answer to our question is no. However, the following question remains open.

QUESTION 3. Suppose $\alpha: \operatorname{Isom}(\mathbb{Q U}) \curvearrowright E$ is a continuous affine action on a reflexive Banach space E. Does $\alpha$ necessarily have a fixed point?

The difference with Theorem 26 here is that the action is not required to be isometric and thus the linear part $\pi$ could map onto an unbounded subgroup of the general linear group GL $(E)$. 


\section{Acknowledgements}

I would like to thank a number of people for helpful conversations and other aid during the preparation of the paper. They include F. Baudier, B. Braga, J. Galindo, G. Godefroy, W. B. Johnson, N. Monod, A. Naor, Th. Schlumprecht and A. Thom. The research was partially supported by a Simons Foundation Fellowship (Grant \#229959) and by the NSF (DMS 1201295 \& DMS 1464974).

\section{References}

[1] I. Aharoni, 'Every separable metric space is Lipschitz equivalent to a subset of $c_{0}$ ', Israel J. Math. 19 (1974), 284-291.

[2] I. Aharoni, 'Uniform embeddings of Banach spaces', Israel J. Math. 27 (1977), 174-179.

[3] I. Aharoni, B. Maurey and B. S. Mityagin, 'Uniform embeddings of metric spaces and of Banach spaces into Hilbert spaces', Israel J. Math. 52(3) (1985), 251-265.

[4] L. Alaoglu and G. Birkhoff, 'General ergodic theorems', Ann. of Math. (2) 41 (1940), 293-309.

[5] F. Albiac and N. Kalton, Topics in Banach Space Theory (Springer, New York, 2006).

[6] U. Bader, T. Gelander and N. Monod, 'A fixed point theorem for $L^{1}$, , Invent. Math. 189(1) (2012), 143-148.

[7] U. Bader, C. Rosendal and R. Sauer, 'On the cohomology of weakly almost periodic group representations’, J. Topol. Anal. 6(2) (2014), 153-165.

[8] M. E. B. Bekka, P.-A. Chérix and A. Valette, 'Proper affine isometric actions of amenable groups', in Novikov Conjectures, Index Theorems and Rigidity, Vol. 2 (Oberwolfach 1993), London Mathematical Society Lecture Note Series, 227 (Cambridge University Press, Cambridge, 1995), 1-4.

[9] B. Bekka, P. de la Harpe and A. Valette, Kazhdan's Property (T), New Mathematical Monographs, 11 (Cambridge University Press, Cambridge, 2008).

[10] I. Ben Yaacov, A. Berenstein and S. Ferri, 'Reflexive representability and stable metrics', Math. Z. 267 (2011), 129-138.

[11] I. Benyamini and J. Lindenstrauss, Geometric Nonlinear Functional Analysis, Vol. 1, (American Mathematical Society, Providence, Rhode Island, 2000).

[12] B. Braga, 'On weaker notions of nonlinear embeddings between Banach spaces', Preprint, 2016.

[13] B. Braga, 'Coarse and uniform embeddings', J. Funct. Anal. 272(5) (2017), 1852-1875.

[14] B. Braga, 'Asymptotic structure and coarse Lipschitz geometry of Banach spaces', Stud. Math. 237(1) (2017), 71-97.

[15] J. Bretagnolle, D. Dacunha-Castelle and J.-L. Krivine, 'Fonctions de type positif sur les espaces $L^{p}$, C. R. Acad. Sci. Paris 261 (1965), 2153-2156. (French).

[16] J. Bretagnolle, D. Dacunha-Castelle and J.-L. Krivine, 'Lois stables et espaces $L^{p}$ ', Ann. Inst. H. Poincaré Sect. B (N.S.) 2 (1965/1966), 231-259. (French).

[17] N. Brown and E. Guentner, 'Uniform embedding of bounded geometry spoaces into reflexive Banach space', Proc. Amer. Math. Soc. 133(7) (2005), 2045-2050.

[18] P.-A. Chérix, M. Cowling, P. Jolissant, P. Julg and A. Valette, Groups with the Haagerup Property: Gromov's a-T-Menability, (Basel, Birkhäuser, 2001).

[19] J. A. Clarkson, 'Uniformly convex spaces', Trans. Amer. Math. Soc. 40 (1936), 396-414. 
[20] Y. de Cornulier, R. Tessera and A. Valette, 'Isometric group actions on Hilbert spaces: growth of cocycles', GAFA, Geom. Funct. Anal. 17 (2007), 770-792.

[21] W. J. Davis, T. Figiel, W. B. Johnson and A. Pełczyński, 'Factoring weakly compact operators', J. Funct. Anal. 17 (1974), 311-327.

[22] P. Enflo, 'Banach spaces which can be given an equivalent uniformly convex norm', Israel J. Math. 13 (1972), 281-288.

[23] M. Fabian, P. Habala, P. Hájek, V. Montesinos and V. Zizler, 'Banach space theory. The basis for linear and nonlinear analysis', in CMS Books in Mathematics/Ouvrages de Mathématiques de la SMC (Springer, New York, 2011).

[24] V. Ferenczi, 'A uniformly convex hereditarily indecomposable Banach space', Israel J. Math. 102 (1997), 199-225.

[25] J. Gervirtz, 'Stability of isometries on Banach spaces', Proc. Amer. Math. Soc. 89 (1983), 633-636.

[26] A. Grothendieck, 'Critères de compacité dans les espaces fonctionnels généraux', Amer. J. Math. 74 (1952), 168-186.

[27] P. M. Gruber, 'Stability of isometries', Trans. Amer. Math. Soc. 245 (1978), 263-277.

[28] E. Guentner and J. Kaminker, 'Exactness and uniform embeddability of discrete groups', J. Lond. Math. Soc. (2) 70 (2004), 703-718.

[29] U. Haagerup, 'An example of a nonnuclear $C^{*}$-algebra, which has the metric approximation property', Invent. Math. 50 (1978), 279-293.

[30] U. Haagerup and A. Przybyszewska, 'Proper metrics on locally compact groups, and proper affine isometric actions on Banach spaces', Preprint, 2006, arXiv:0606794.

[31] P. de la Harpe, 'Moyennabilité du groupe unitaire et propriété $\mathrm{P}$ de Schwartz des algèbres de von Neumann', in Algèbres d'opérateurs (Sém., Les Plans-sur-Bex, 1978), Lecture Notes in Mathematics, 725 (Springer, Berlin, 1979), 220-227.

[32] W. Herer and J. P. R. Christensen, 'On the existence of pathological submeasures and the construction of exotic topological groups', Math. Ann. 213 (1975), 203-210.

[33] W. B. Johnson and N. L. Randrianarivony, ' $\ell_{p}(p>2)$ does not coarsely embed into a Hilbert space', Proc. Amer. Math. Soc. 134(4) (2006), 1045-1050.

[34] N. J. Kalton, 'Coarse and uniform embeddings into reflexive spaces', Quart. J. Math. 58(3) (2007), 393-414.

[35] N. J. Kalton, 'The non-linear geometry of Banach spaces', Rev. Mat. Complut. 21(1) (2008), $7-60$.

[36] N. J. Kalton, 'The uniform structure of Banach spaces', Math. Ann. 354(4) (2012), 1247-1288.

[37] A. S. Kechris and C. Rosendal, 'Turbulence, amalgamation and generic automorphisms of homogeneous structures', Proc. Lond. Math. Soc. (3) 94(2) (2007), 302-350.

[38] J.-L. Krivine and B. Maurey, 'Espaces de Banach stables', Israel J. Math. 39(4) (1981), 273-295.

[39] W. Lusky, 'The Gurarij spaces are unique', Arch. Math. 27 (1976), 627-635.

[40] M. G. Megrelishvili, 'Every semitopological semigroup compactification of the group $H_{+}[0,1]$ is trivial', Semigroup Forum 63(3) (2001), 357-370.

[41] M. G. Megrelishvili, 'Operator topologies and reflexive representability', in Nuclear Groups and Lie Groups (Madrid, 1999), Research and Exposition in Mathematics, Vol. 24 (Heldermann, Lemgo, 2001), 197-208.

[42] M. Mendel and A. Naor, 'Euclidean quotients of finite metric spaces', Adv. Math. 189 (2004), 451-494.

[43] M. Mendel and A. Naor, 'Metric cotype', Ann. of Math. (2) 168 (2008), 247-298. 
[44] N. Monod, Continuous Bounded Cohomology of Locally Compact Groups, Lecture Notes in Mathematics, 1758 (Springer, Berlin Heidelberg, 2001).

[45] E. H. Moore, 'On properly positive Hermitian matrices', Bull. Amer. Math. Soc. (N.S.) 23(59) (1916), 66-67.

[46] A. Naor, 'Uniform nonextendability from nets', C. R. Acad. Sci.- Series I - Mathématique 353(11) (2015), 991-994.

[47] A. Naor and Y. Peres, ' $L_{p}$ compression, traveling salesmen, and stable walks', Duke Math. J. 157(1) (2011), 53-108.

[48] E. Odell and T. Schlumprecht, 'The distortion problem', Acta Math. 173 (1994), 259-281.

[49] V. Pestov, 'A theorem of Hrushovski-Solecki-Vershik applied to uniform and coarse embeddings of the Urysohn metric space', Topol. Appl. 155(14) (2008), 1561-1575.

[50] G. Pisier, 'Martingales with values in uniformly convex spaces', Israel J. Math. 20 (1975), 326-350.

[51] N. L. Randrianarivony, 'Characterization of quasi-Banach spaces which coarsely embed into a Hilbert space', Proc. Amer. Math. Soc. 134(5) (2006), 1315-1317.

[52] Y. Raynaud, 'Espaces de Banach superstables, distances stables et homéomorphismes uniformes, (French. English summary) [Superstable Banach spaces, stable distances and uniform homeomorphisms]', Israel J. Math. 44(1) (1983), 33-52.

[53] J. Roe, Lectures on Coarse Geometry, University Lecture Series, 31 (American Mathematical Society, Providence, RI, 2003).

[54] S. Rolewicz, Metric Linear Spaces (Reidel, Dordrecht, 1985).

[55] C. Rosendal, 'A topological version of the Bergman property', Forum Math. 21(2) (2009), 299-332.

[56] C. Rosendal, 'Finitely approximable groups and actions part I: the Ribes-Zalesskiu property', J. Symbolic Logic 76(4) (2011), 1297-1306.

[57] C. Rosendal, 'Coarse geometry of topological groups', book manuscript 2017.

[58] C. Ryll-Nardzewski, 'Generalized random ergodic theorems and weakly almost periodic functions', Bull. Acad. Polon. Sci. Sér. Sci. Math. Astronom. Phys. 10 (1962), 271-275.

[59] F. M. Schneider and A. Thom, 'On Følner sets in topological groups', Preprint, 2016, arXiv: 1608.08185 .

[60] A. I. Shtern, 'Compact semitopological semigroups and reflexive representability of topological groups', Russ. J. Math. Phys. 2(1) (1994), 131-132.

[61] M. Simonnet, Measures and Probabilities (Springer, New york, 1996).

[62] S. Solecki, 'Extending partial isometries', Israel J. Math. 150 (2005), 315-332. 